\title{
1 High Accuracy Schemes for DNS and Acoustics
}

\author{
T. K. Sengupta, ${ }^{1}$ S. K. Sircar, ${ }^{2}$ and A. Dipankar ${ }^{1}$
}

Received May 1, 2004; accepted (in revised form) January 7, 2005

\begin{abstract}
High-accuracy schemes have been proposed here to solve computational acoustics and DNS problems. This is made possible for spatial discretization by optimizing explicit and compact differencing procedures that minimize numerical error in the spectral plane. While zero-diffusion nine point explicit scheme has been proposed for the interior, additional high accuracy one-sided stencils have also been developed for ghost cells near the boundary. A new compact scheme has also been proposed for non-periodic problems - obtained by using multivariate optimization technique. Unlike DNS, the magnitude of acoustic solutions are similar to numerical noise and that rules out dissipation that is otherwise introduced via spatial and temporal discretizations. Acoustics problems are wave propagation problems and hence require Dispersion Relation Preservation (DRP) schemes that simultaneously meet high accuracy requirements and keeping numerical and physical dispersion relation identical. Emphasis is on high accuracy than high order for both DNS and acoustics. While higher order implies higher accuracy for spatial discretization, it is shown here not to be the same for time discretization. Specifically it is shown that the 2nd order accurate Adams-Bashforth (AB) - scheme produces unphysical results compared to first order accurate Euler scheme. This occurs, as the AB-scheme introduces a spurious computational mode in addition to the physical mode that apportions to itself a significant part of the initial condition that is subsequently heavily damped. Additionally, AB-scheme has poor DRP property making it a poor method for DNS and acoustics. These issues are highlighted here with the help of a solution for (a) Navier-Stokes equation for the temporal instability problem of flow past a rotating cylinder and (b) the inviscid response of a fluid dynamical system excited by simultaneous application of acoustic, vortical and entropic pulses in an uniform flow. The last problem admits analytic solution for small amplitude pulses and can be used to calibrate different methods for the treatment of non-reflecting boundary conditions as well.
\end{abstract}

KEY WORDS: Computational acoustics; DNS; compact difference schemes; dispersion relation preservation schemes; time discretization.

\footnotetext{
${ }^{1}$ Department of Aerospace Engineering, I.I.T. Kanpur, U.P. 208016, India. E-mail: tksen@ iitk.ac.in

${ }^{2}$ Department of Mathematics, I.I.T. Kanpur, U.P. 208016, India.
} 


\section{INTRODUCTION}

With powerful computers and newer methods it is now routine to solve the governing Navier-Stokes equation resolving all the scales for turbulent flows by DNS at moderate Reynolds numbers. In this context compact and other higher-order schemes are finding more and more applications. Similarly, in wave propagation problems one solves hyperbolic partial differential equations and such solutions are required to be accurate in the far field and for long time periods. These requirements demand that the adopted numerical method be highly accurate and dispersion error free. Lighthill [1] has discussed the problems of computational aero-acoustics (CAA) with respect to these issues.

The compact schemes, based on Padé approximation, offer highaccuracy approximations to differential and integral operators using compact implicit stencils. Some of the early works in this field are reported in [2-4].

For DNS of incompressible flows, it is important to compute flows with large directional convection of vortical structures. Thus, DNS requires capturing high amplitude signals without suffering numerical instabilities. This instability may be caused due to linear instability, error accumulation due to aliasing and/or non-linear instabilities. While using compact schemes, it is thus quite common to add numerical dissipation via upwinding during discretization [5-7] or filtering $[4,8]$ the solution after each time step. The basic idea of adding algebraically a dissipation term is equivalent to providing a negative feedback. Thus, if one uses 2 nd derivative as numerical dissipation then it is strictly added, while for the 4th derivative term the dissipation term has to be subtracted. Quite often, in the literature, this has been stated simply as "adding numerical dissipation".

In contrast, solving acoustics problems involve capturing weak signals that are hard to distinguish from numerical errors. Thus, one of the major consideration is that one should not add numerical dissipation that would remove useful high frequency - high wave number parts of the signal. In a major work Tam and Webb [9] discussed this and the issue of using DRP schemes for computational acoustics. Unlike in DNS, acoustic signal propagation can be treated as a linear phenomenon in the absence of attendant flow instabilities. If one works in the physical plane, there are no problems of aliasing error and the main concern in computational acoustics is one of accuracy and avoiding spurious reflections from computational boundaries. High accuracy requirements can be achieved by optimizing the finite difference approximations of derivatives in the wave number space, as the truncation error is minimized in [4,6,7,9,10]. In Tam and Webb [9] this has been separately for the spatial and temporal derivatives using explicit 
schemes. In the other references this has been performed for spatial derivatives only using compact schemes. In the present exercise the optimization process would be extended to non-periodic problems for both the explicit and a compact schemes.

In many researches, disproportionate amount of attention has been paid on the accuracy of spatial discretization in comparison to temporal discretization. In many applications, first order accurate Euler time integration is used for DNS and computational acoustics. In contrast, in [9] time integration is performed by an optimized stencil that is $O\left(\Delta t^{3}\right)$ accurate. It is usual to expect that a choice of higher order time integration schemes will help achieve higher accuracy and allow taking larger time steps. Explicit higher order time integration schemes are also commonly in use for reactive flow computations [11] and geophysical fluid dynamics [12]. For example, in weather predictions using inviscid equations, three time-level leapfrog marching scheme is used and then the numerical procedure would bring in two amplification factors. For example, when this time integration strategy is used for integrating one-dimensional advection equation, both the amplification factors $\left(G_{1}\right.$ and $\left.G_{2}\right)$ indicate neutral behavior, but with phase error (see [13] for details). In this paper, the following notations have been used to express the amplification factors. While $G_{1}$ and $G_{2}$ denote the amplification factors for physical and computational modes, subsequently we have used $G^{(2)}$ to indicate the amplification factor with Euler time integration, where the superscript within brackets denote the order of spatial discretization.

Weather prediction with leapfrog time marching, decorrelates with time due to aliasing error, phase error and other effects due to nonlinearity. Haltiner and Williams [13] have shown that one component of the solution (called the physical mode) corresponding to $G_{1}$ approaches exact solution, while the second component of the solution (called the computational mode) corresponding to $G_{2}$, approaches zero as $\Delta x$ and $\Delta t$ are allowed to approach zero. It is also noted in [13] that the computational mode alternates in sign with every time step and propagates in the opposite direction of the exact solution. Thus, the second mode is a spurious one would be a source of numerical error. Similarly, the time integration strategy used in [9] has four modes- out of which three 110 schemes may lead to numerical instability due to poor property of any one 111 of the spurious modes. Fig. 3(b) of [9] clearly shows that $\omega \Delta t$ has to be 112 chosen less than 0.4 to avoid this instability, negating the advantages of 113 higher order time integration scheme. In fact, the physical mode also shows 114 strong attenuation beyond this value of $\omega \Delta t$, while the other two modes 115 are severely damped. 
Lilly [14], while examining time advancement schemes for simplified form of barotropic vorticity equation noted that the second order AdamsBashforth $(\mathrm{AB})$ scheme performs the best with respect to efficiency and accuracy. For a typical time evolution equation,

$$
\frac{d f}{d t}=F(f, t)
$$

121 the $\mathrm{AB}$-scheme is given by,

$$
f\left(t_{n+1}\right)=f\left(t_{n}\right)+\frac{\Delta t}{2}\left[3 F\left(t_{n}\right)-F\left(t_{n-1}\right)\right] .
$$

For this scheme, it is noted in [13] that the computational mode is heavily damped and the physical mode has to be kept from becoming unstable by keeping $\Delta t$ small. It is also stated that -the Adams-Bashforth scheme is suitable unless the period of integration is lengthy [13]. Despite this cautionary note, this scheme is finding application in many researches

128 in the so called DNS that would require solving governing equations for 129 long time. For example, among innumerable references, it has been used in 130 finite difference methods of solving Navier-Stokes equation in [15], [16] 131 for channel flow; in [17] for flow over a wavy wall; in [18] for jet flows; 132 in [19] for boundary layer instability; in [20] for free surface channel 133 flow and in [21] for LES. It has even been used for spectral calculations 134 in [22]. Hence a detailed analysis of this scheme is warranted. However, none of these references used compact schemes that are proving to be very useful for DNS. Thus, it is also necessary to analyze AB-time integration scheme for its suitability for DNS and acoustics when used with compact schemes. We also explore the four-stage Runge-Kutta time integration scheme (RK4) that is often used for high accuracy computing.

The paper is structured in the following manner. In the next section we discuss and develop various explicit and implicit schemes for spatial discretization. In Sec. 2 various time discretization schemes are analyzed with the help of one- dimensional convection equation. In Sec. 3, two examples drawn from acoustics and flow instability are shown to highlight various issues discussed herein.

\section{HIGH ACCURACY SCHEMES FOR SPATIAL DERIVATIVES}

In compact schemes, on a uniform grid of spacing $h=\Delta x$, the first derivative $u^{\prime}$ is obtained from the solution of the following linear algebraic equation: 
This is an implicit linear algebraic equation involving the derivatives and function values at different nodes. If $[A]$ is an identity matrix, then we have corresponding explicit schemes. For the purpose of analysis, the above equation is rewritten as,

$$
u^{\prime}=[C] u .
$$

This method of evaluation of first derivative can be represented in the spectral plane [7] by $u^{\prime}\left(x_{j}\right)=\int i k_{e q} \hat{U}(k) e^{i k x_{j}} d k$, where

$$
i k_{e q}\left(x_{j}\right)=\sum_{l=1}^{N} C_{l j} e^{i k\left(x_{l}-x_{j}\right)} .
$$

This general method of characterizing any discretization technique in the spectral plane was introduced in [7] and provides a means for full domain analysis simultaneously. Such an approach becomes very relevant to evaluate various boundary closure schemes. Some optimal globally stable schemes were introduced in [7] with the help of this method. Different numerical schemes have different estimates of $k_{e q}$ and it is in general a complex quantity. The imaginary part of $k_{e q}$ represents numerical dissipation when it is negative. A spatial discretization scheme, that has a positive imaginary part of $k_{e q}$ at a point, locally contributes to numerical instability as it is equivalent to adding anti-diffusion.

The developed methodology in [7] can form the basis of optimization to develop new high accuracy schemes for non-periodic problems. Essential ideas for periodic problems or only for the interior stencils of a compact scheme for non-periodic problem have been discussed in $[4,6,7,10]$ and a brief account is added here for ease of understanding. The following constrained minimization problem, whose solution would provide a high accuracy scheme with improved resolution is attempted whereby one minimizes

$$
E(., .)=\sum_{l=1}^{N} e_{l}=\sum_{l=1}^{N} \int\left|L_{h}^{l}(k h)-L^{l}(k h)\right|^{2} U^{2}(k) d k .
$$

Here $L^{l}(k h)=i k h$ and $L_{h}^{l}(k h)=\sum_{j=1}^{N} C_{l j}\left(R_{l j}+i I_{l j}\right)$ are the exact and numerical differential operators operating on the Laplace transform of the initial condition of the function. Arguments on the left-hand side of (6) are the parameters over which the problem is minimized. In optimizing the stencil, we use $U(k)=1$, so that we are seeking a conservative estimate with respect to a white noise or Dirac delta excitation of the system. In [10] this optimization was performed for periodic one-dimensional wave 
185 equation with a particular type of band-limited spectra of initial data. The objective function for the $l$ th node can be expressed as,

$$
e_{l}=\int_{k_{m}}^{k_{m}}\left|\sum_{j=1}^{N} C_{l j} R_{j l}+i \sum_{j=1}^{N}\left(C_{l j} I_{l j}-k h\right)\right|^{2} d k
$$

The above can be further simplified to

$$
e_{l} /[\pi(N-1)]=\frac{2 \pi^{2}}{3}+\sum_{j \neq l}^{j=N} \frac{4 C_{l j}}{j-l}(-1)^{(j-l)}+2 C_{l l}^{2}+\sum_{j \neq l}^{j=N} C_{l j}^{2} .
$$

This $L_{2}$ - norm for error for the approximation of first derivative is dependent on the property of $C$ matrix i.e. on $A$ and $B$ matrices in Eq. (3). The first term in the above equation, is contributed by the exact differential operator and is always positive. The third and fourth terms are also positive and cannot reduce the error norm, except the fact that $C_{l l}$ can be made identically equal to zero. This is the case for explicit central difference schemes and they can be termed as low error schemes as compared to equivalent upwind schemes. The off-diagonal terms of $C$ matrix can reduce error through the second term in (8). As $C_{l j}$ is scaled by $(l-j)$, most of the contributions would come from the immediate neighboring points of the diagonal. The contributions coming from $j=l \pm 1$ and $j=l \pm 2$ are $4\left[C_{l l-1}-C_{l l+1}\right]$ and $2\left[C_{l l-2}-C_{l l+2}\right]$, respectively. For example, one can estimate the error for 2 nd -order and 4 th -order central differencing schemes as equal to $\left(\left(2 \pi^{2} / 3\right)-(3 / 2)\right)$ and $\left(\left(2 \pi^{2} / 3\right)-(319 / 72)\right)$ respectively.

Here we intend to develop a high accuracy optimized compact scheme for non-periodic problems. For this purpose we intend using the following stencil for the interior point,

$$
\alpha u_{l-1}^{\prime}+u_{l}^{\prime}+\alpha u_{l+1}^{\prime}=\frac{b}{4 h}\left(u_{l+2}-u_{l-2}\right)+\frac{a}{2 h}\left(u_{l+1}-u_{l-1}\right) .
$$

For non-periodic problems, one would require special one-sided boundary stencils as the ones used in [7]. For the first and second points of the domain they are given by,

$$
u_{1}^{\prime}=\frac{1}{2 h}\left(-3 u_{1}+4 u_{2}-u_{3}\right)
$$

$$
u_{2}^{\prime}=\frac{1}{h}\left[\left(\frac{2 \gamma_{2}}{3}-\frac{1}{3}\right) u_{1}-\left(\frac{8 \gamma_{2}}{3}+\frac{1}{2}\right) u_{2}+\left(4 \gamma_{2}+1\right) u_{3}-\left(\frac{8 \gamma_{2}}{3}+\frac{1}{6}\right) u_{4}+\frac{2 \gamma_{2}}{3} u_{5}\right]
$$


Similarly, one can write down the boundary closure schemes for $j=N$ and $j=N-1$ using $\gamma_{N-1} \cdot \gamma_{j}$ are the free parameters chosen for $j=2$ and $j=N-1$ independently. Eqs. (9)-(11) would assist one in compiling the $C$ matrix and thus it is easy to see that $E$ is a function of $\left(\alpha, a, b, \gamma_{2}, \gamma_{N-1}\right)$. $E$ has to be optimized subject to the compatibility condition: $1+2 \alpha=a+$ $b$, that ensures at least second-order accuracy. To search for the optimum, multivariate evolutionary optimization technique of [23] is used that gave the following values for a choice of $N=30$ as,

$$
a=1.546277, \quad b=0.329678, \quad \gamma_{2}=-0.025 \quad \text { and } \quad \gamma_{N-1}=0.09 .
$$

These parameter values and the optimum does not change when $N$ is increased further. Following the convention in [7], we refer to this as OUCS4 scheme in the subsequent discussion.

Following the above procedure, one can also develop a high accuracy explicit stencils for the first derivative. In [9], a fourth order accurate seven point central stencil was designed for computational acoustics problem. In the following, we similarly develop a nine point stencil for the evaluation of first derivative explicitly:

$$
\begin{aligned}
u_{l}^{\prime}= & \frac{a_{0}}{2 h}\left(u_{l+1}-u_{l-1}\right)+\frac{b_{0}}{4 h}\left(u_{l+2}-u_{l-2}\right) \\
& +\frac{d_{0}}{6 h}\left(u_{l+3}-u_{l-3}\right)+\frac{e_{0}}{8 h}\left(u_{l+4}-u_{l-4}\right) .
\end{aligned}
$$

Equating the successive terms of the Taylor series, the following one parameter relations are obtained in terms of $a_{0}$ as: $b_{0}=12 / 5-2 a_{0} ; d_{0}=$ $\left(45 a_{0}-64\right) / 35$ and $e_{0}=\left(3-2 a_{0}\right) / 7$. When the corresponding spectral error is minimized one obtains the optimum for $a_{0}=1.66631451979287$. For actual usage of this scheme, one would require boundary stencils for four layers of points those have to be one-sided. For example, one could obtain the derivative at $l=1$ by,

$$
u_{1}^{\prime}=\frac{1}{h} \sum_{j=1}^{9} a_{j} u_{j},
$$

where all the coefficients are written in terms of $a_{1}$ by equating coefficients of the Taylor series on either side as, $a_{2}=-\left(8 a_{1}+481 / 35\right) ; a_{3}=$ $28 a_{1}+621 / 80 ; a_{4}=-\left(56 a_{1}+2003 / 15\right) ; a_{5}=70 a_{1}+691 / 4 ; a_{6}=-\left(56 a_{1}+\right.$ $141) ; a_{7}=\left(28 a_{1}+2143 / 30\right) ; a_{8}=-\left(8 a_{1}+103 / 5\right)$ and $a_{9}=a_{1}+363 / 140$. Once again optimization provides one with $a_{1}=-2.62538939007719$ for least error. The same procedure is repeated here for the points at $l=2,3$ and 4 as well. For brevity, we will call these collectively as the SS- scheme. 

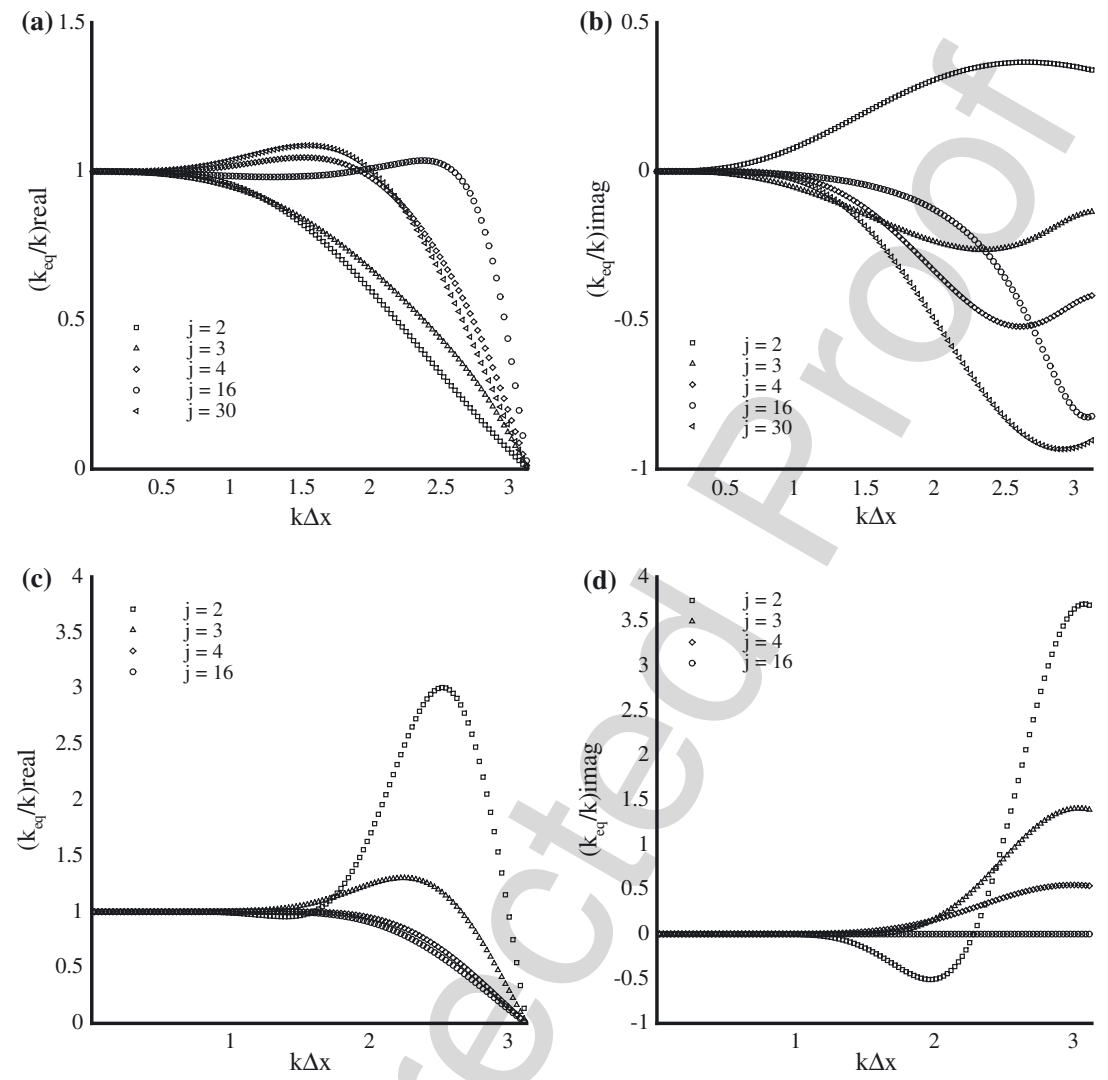

Fig. 1. $k_{e q} / k$ for the first derivative at different nodes evaluated using OUCS4 (Figures (a) and (b)) and SS (Figures (c) and (d)) schemes.

In Fig. 1, the real and imaginary parts of $k_{e q} / k$ is shown for OUCS4 and the above explicit schemes for different nodes, using the methodology of [7]. The real part reveals the superior spectral accuracy of OUCS4 up to $k h=2.65$ as compared to 2.2 for the scheme given in [10] that was found to have largest spectral resolution among the known schemes for periodic problems. The imaginary part by itself reveals anti-diffusion for near boundary points and is not directly suitable for use. To obtain uniform attenuation for all wave numbers and no instabilities we introduce fourth order dissipation to achieve negative feedback stabilization.

Since the solution of Navier-Stokes equation uses Dirichlet boundary conditions in the non-periodic direction the properties of the scheme for the points at $l=1$ and $l=N$ are not relevant. In Fig. 1, the real and 
261 imaginary parts of $k_{e q} / k$ are also plotted for the SS- scheme. The inte262 rior point stencil being symmetric, it is non-dissipative and hence $k_{e q} / k$ is 263 purely real. The boundary stencils are not so and would stabilize or desta264 bilize the discrete equation depending on its sign.

These two optimum schemes will be assessed along with the OUCS3 scheme of [7] and a third-order scheme described in [24].

267

268

269

270

271

272

273

274

275

276

277

278

279

280

281

282

283

284

285

286

287

\section{TEMPORAL DISCRETIZATION SCHEMES}

Temporal discretization in conjunction with spatial discretization can be studied only with respect to standard equations. For this purpose, we consider the propagation problem given by the one-dimensional convection equation,

$$
\frac{\partial u}{\partial t}+c \frac{\partial u}{\partial x}=0
$$

for which the initial solution $u_{o}(x)$ travels to the right with the phase speed $c$. For the numerical solution of the wave equation, we identify it as

such that the initial solution is given by,

$$
u_{m}^{o}=\int A_{o}(k) e^{i k x_{m}} d k
$$

The following time integration schemes to be used in conjunction with different spatial schemes are described briefly. First, we define the various properties of the time integration schemes when used with some standard differencing schemes for spatial derivatives.

\subsection{Euler Time Integration Scheme}

For Euler time integration and second order central differencing for the spatial derivative, if we define the CFL number by $N_{c}=\frac{c \Delta t}{\Delta x}=\frac{\omega \Delta t}{k \Delta x}$, then the amplification factor $G^{(2)}(k)=\frac{B\left(k, t^{n+1}\right)}{B\left(k, t^{n}\right)}$ is,

$$
G^{(2)}(k)=\left(1+N_{c}^{2} \sin ^{2} k \Delta x\right)^{1 / 2} e^{-i \beta_{2}},
$$


288 where $\tan \beta_{2}=N_{c} \sin k \Delta x$. The general solution at any arbitrary time is,

$$
\begin{aligned}
u_{m}^{n} & =\int A_{0}(k)\left[G^{(2)}(k)\right]^{n} e^{i k x_{m}} d k \\
& =\int A_{0}(k)\left[1+N_{c}^{2} \sin ^{2} k \Delta x\right]^{\frac{n}{2}} e^{i\left(k x_{m}-n \beta_{2}\right)} d k .
\end{aligned}
$$

291 Thus, $\beta_{2}$ gives a measure of the phase speed of the numerical scheme that is given by $c_{N}=\frac{\beta_{2}}{k \Delta t}$ while the scaled phase speed is $\frac{c_{N}}{c}=\frac{\beta_{2}}{\omega \Delta t}$.

If we replace the second order spatial discretization scheme by fourthorder central scheme, as given by

$$
\left(\frac{\partial u}{\partial x}\right)_{m}=\frac{1}{12 \Delta x}\left[-u_{m+2}+8 u_{m+1}-8_{m-1}+u_{m-2}\right]
$$

then one obtains the following amplification factor $G^{(4)}(k)=1-i \frac{N_{c}}{3}[4-$ $\cos k \Delta x] \sin k \Delta x$ and the general solution at any arbitrary time is

$$
u_{m}^{n}=\int A_{0}(k)\left[1+\frac{N_{c}^{2}}{9}(4-\cos k \Delta x)^{2} \sin ^{2} k \Delta x\right]^{\frac{n}{2}} e^{i\left(k x_{m}-n \beta_{4}\right)} d k,
$$

where $\tan \beta_{4}=\frac{N_{c}}{3}[4-\cos (k \Delta x)] \sin (k \Delta x)$.

It is well known that the above schemes do not produce stable results. This is often rectified by resorting to upwinding, as discussed in [24]. In particular, a third-order upwind scheme is considered here, that calculates

303 the first derivative from the following stencil for Eq. (14):

$$
\frac{\partial u}{\partial x}=\frac{1}{6 \Delta x}\left[u_{m+2}^{n}-2 u_{m+1}^{n}+9 u_{m}^{n}-10 u_{m-1}^{n}+2 u_{m-2}^{n}\right] .
$$

where $L_{2}=2[4-\cos (k \Delta x)] \sin (k \Delta x)$ and $L_{1}=-24 \sin ^{4}\left(\frac{k \Delta x}{2}\right)$. This can be rewritten as

$$
G^{(3)}(k)=1-i \frac{N_{c}}{6}\left(L_{2}+i L_{1}\right),
$$

310 with which one can obtain the expression for numerical phase speed from $311 \tan \left(\beta_{3}\right)=\left(\frac{N_{c} L_{2}}{6+N_{c} L_{1}}\right)$. The general solution at arbitrary time is given by,

$$
\begin{aligned}
& G^{(3)}(k)=\left[\left(1+\frac{N_{c}}{6} L_{1}\right)^{2}+\frac{N_{c}^{2} L_{2}^{2}}{36}\right]^{\frac{1}{2}} e^{-i \beta_{3}} \\
& =\left(\frac{N_{c} L_{2}}{6+N_{c} L_{1}}\right) \text {. The general solution at arbitrary time is given } \\
& u_{m}^{n}=\int A_{0}(k)\left[\left(1+\frac{N_{c}}{6} L_{1}\right)^{2}+\frac{N_{c}^{2} L_{2}^{2}}{36}\right]^{\frac{n}{2}} e^{i\left(k x_{m}-n \beta_{3}\right)} d k
\end{aligned}
$$


313 In Fig. 2 the amplification factor for some of these spatial discretization 314 schemes are shown, with Euler time integration scheme. It is evident that the central schemes, including the SS scheme, are unstable for any time steps chosen. The $3 \mathrm{rd}$ order scheme allows taking a very small $\Delta t$ for stability at small $k$ 's. It allows larger $\Delta t$ for larger values of $k \Delta x$. The third order scheme performs the best among these schemes. The SS stencil for the second point is asymmetric and it shows a range of $k \Delta x$ where the scheme is selectively stable.

In Fig. 3 the contours of the numerical phase speed are plotted. One can note regions where the numerical phase speed is within $5 \%$ tolerance of the exact value. From operational considerations, the contiguous region near the origin is the useful range. The OUCS4 scheme has the best behavior by this yardstick.

For DRP property, the relevant quantity is the group velocity of the schemes that can be evaluated from the numerical dispersion relation, $\omega_{e q}=c_{N} k$, from which the scaled numerical group velocity is evaluated as,

$$
\frac{V_{g N}}{c}=\frac{c_{N}}{c}+\frac{k^{2}}{\omega} \frac{d c_{N}}{d k}=-\frac{1}{N_{c} h} \frac{d \beta_{i}}{d k} .
$$

The right-hand side of the above can be estimated for any combination of spatial and temporal discretization schemes. In Fig. 4, results are graphically displayed in the $(k \Delta x-\omega \Delta t)$ - plane as contour plots. It is seen that among all schemes considered here, OUCS4 scheme performs the

\subsection{Adams-Bashforth Time Integration Scheme}

Application of AB-scheme for time integration of Eq. (14) along with 2nd order central differencing yields the following discrete equation,

339 for which the amplification factors are the roots of the quadratic equation,

$$
(G-1)+i \frac{N_{c}}{2}\left(3-\frac{1}{G}\right) L=0,
$$

341 where $L=\sin (k \Delta x)$. If the roots are indicated by $\lambda_{1}$ and $\lambda_{2}$ then

$$
\begin{gathered}
\lambda_{1}=F e^{i \eta}, \\
\lambda_{2}=H e^{i \Gamma},
\end{gathered}
$$



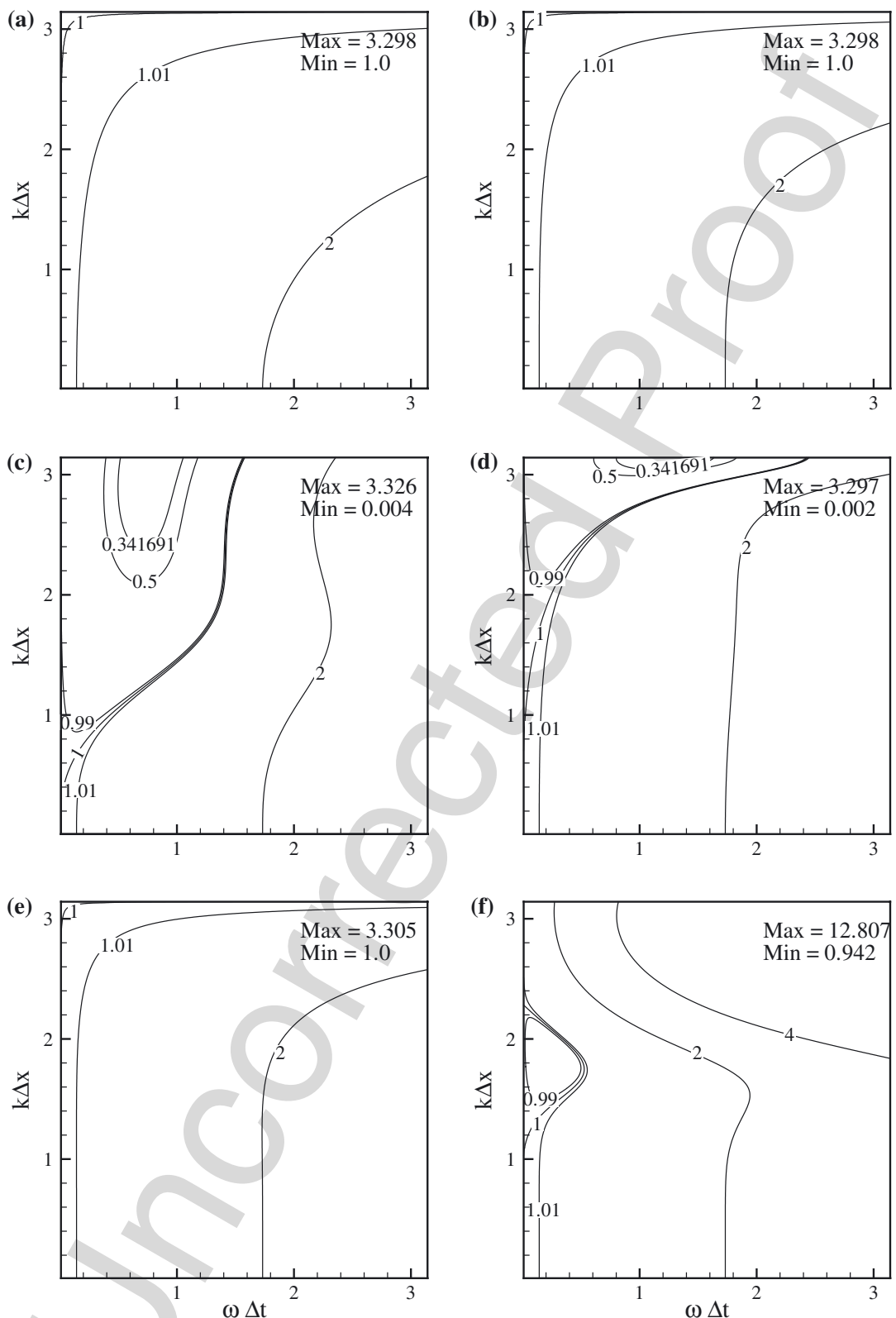

Fig. 2. Amplification factor for solving 1D wave equation with Euler time integration scheme and (a) $C D_{2}$; (b) $C D_{4}$; (c) $U D_{3}$; (d) OUCS4; (e) SS-interior and (f) SS-second scheme for spatial discretization. 

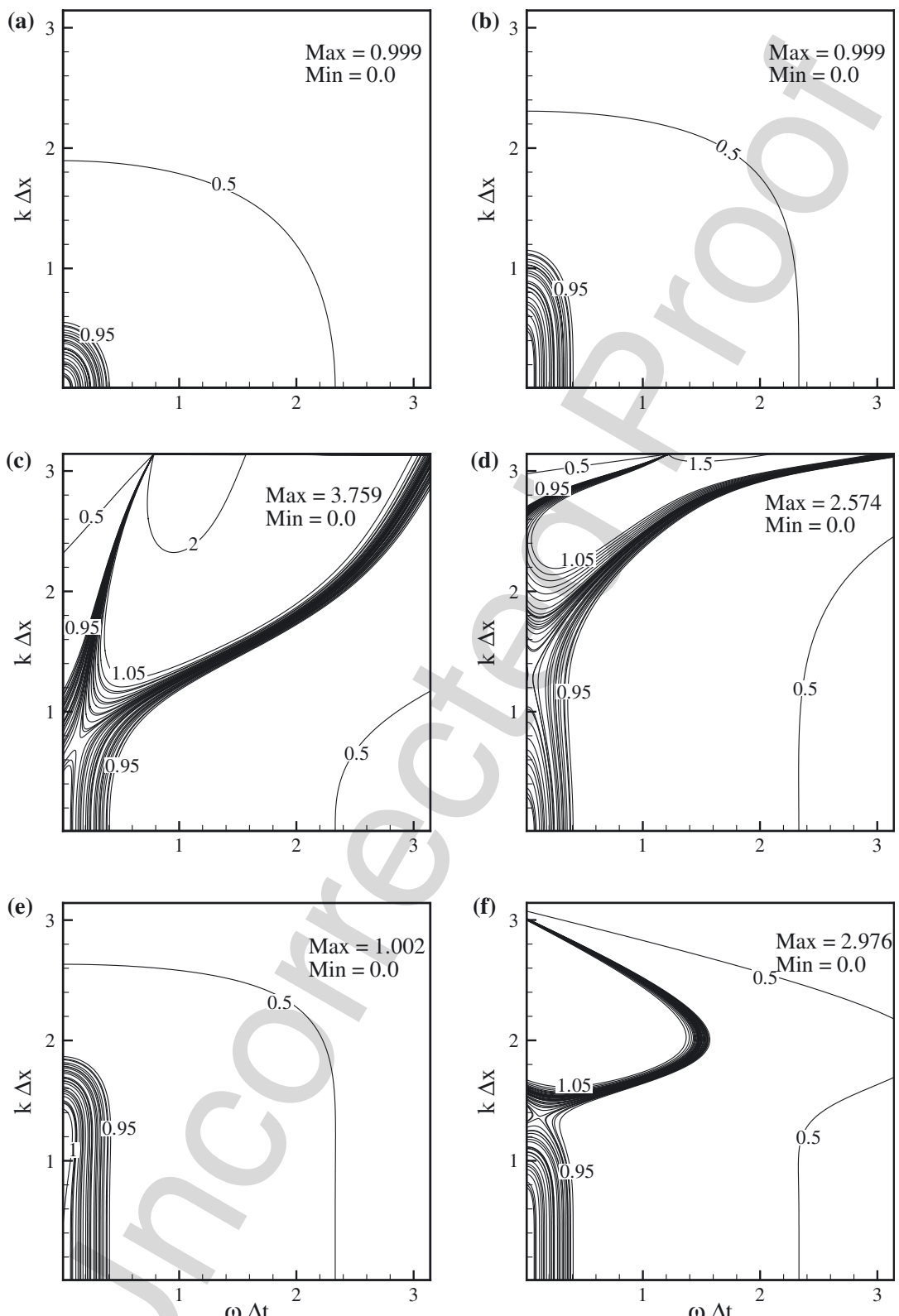

Fig. 3. Scaled numerical phase speed $\left(c_{N} / c\right)$ for solving $1 \mathrm{D}$ wave equation by the schemes indicated in Fig. 2. 

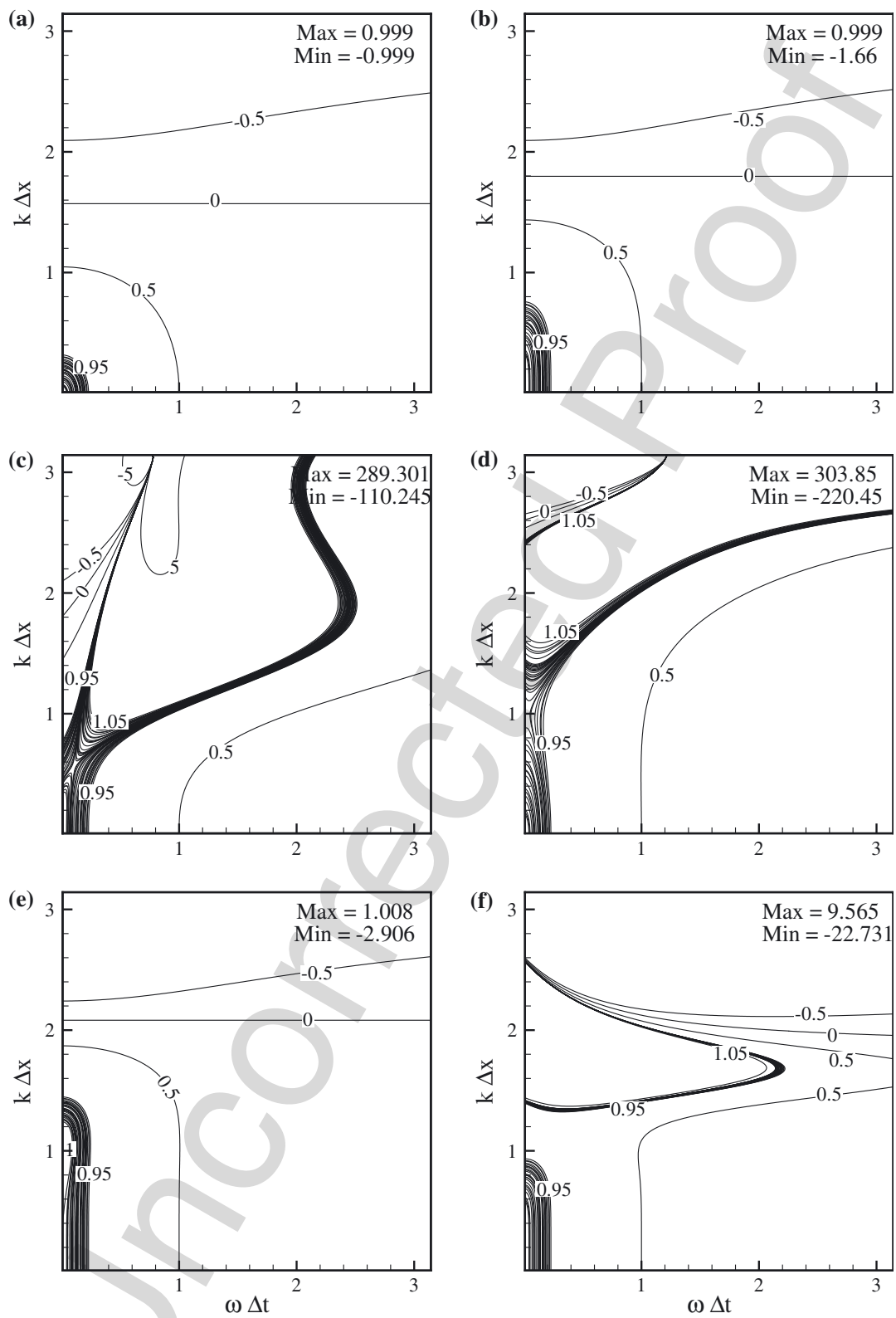

Fig. 4. Scaled numerical group velocity $\left(V_{g N} / c\right)$ for solving $1 \mathrm{D}$ wave equation by the schemes indicated in Fig. 2. 
344 where,

346

$$
\begin{array}{r}
F(k)=\left[C^{2}+D^{2}+2 C D \cos \left(\frac{\xi}{2}-\frac{\bar{\beta}}{2}\right)\right]^{1 / 2}, \\
H(k)=\left[C^{2}+D^{2}-2 C D \cos \left(\frac{\xi}{2}-\frac{\bar{\beta}}{2}\right)\right]^{1 / 2}, \\
C=\frac{1}{2}\left[1+\frac{9}{4}\left(N_{c} L\right)^{2}\right]^{1 / 2},
\end{array}
$$

$$
D=\frac{1}{2}\left[1+\frac{81}{16}\left(N_{c} L\right)^{4}-\frac{7}{2}\left(N_{c} L\right)^{2}\right]^{1 / 4}
$$

$$
\tan (\eta)=\frac{C \sin \left(\frac{\xi}{2}\right)+D \sin \left(\frac{\bar{\beta}}{2}\right)}{C \cos \left(\frac{\xi}{2}\right)+D \cos \left(\frac{\bar{\beta}}{2}\right)},
$$

$$
\tan (\Gamma)=\frac{C \sin \left(\frac{\xi}{2}\right)-D \sin \left(\frac{\bar{\beta}}{2}\right)}{C \cos \left(\frac{\xi}{2}\right)-D \cos \left(\frac{\bar{\beta}}{2}\right)} .
$$

And the general solution is,

$$
u_{m}{ }^{n}=\int M(k)[F]^{n} e^{i\left(k x_{m}+n \eta\right)} d k+\int N(k)[H]^{n} e^{i\left(k x_{m}+n \Gamma\right)} d k .
$$

355 In Eq. (25) the first part of the solution is the physical mode and the sec356 ond part is the computational mode. Ideally one expects the computational 357 mode to contribute by negligible amount. In the above expression $F$ and $358 H$ constitute the time dependent part. The multiplicative constants $M$ and $359 N$ in Eq. (25) can be evaluated from the conditions at $t=0$ (given by Eq. 360 (16) ) and at $t=\Delta t$ (obtained from Eq. (17) for $n=1$ ). Substitution and 361 simplification yields,

$$
\begin{aligned}
& M(k)=A_{0} \frac{1-i N_{c} L-H e^{i \Gamma}}{F e^{i \eta}-H e^{i \Gamma}}, \\
& N(k)=A_{0} \frac{-1+i N_{c} L+F e^{i \eta}}{F e^{i \eta}-H e^{i \Gamma}} .
\end{aligned}
$$


As $M+N=A_{0}$, it implies that $M$ and $N$ distributes the initial condition between the physical and computational modes. The overall performance portrait of this time integration scheme is shown in Fig. 5, where $F, H, M$ and $N$ are plotted for the $C D_{2}$ spatial discretization scheme for Eq. (14). From Fig. 5(a) and (b), the reason for the nomenclature of the physical and computational modes is apparent. As the computational mode is severely attenuated, it is noted in the literature that this mode does not contribute after few time steps. While this is true, Fig. 5(c) and (d) indicate another important aspect that has been overlooked earlier. The physical mode carries all the information of the initial condition (where it is equal to one) only along two lines and everywhere else it is either overor under-estimated. Wherever it is under-estimated, for small $k$ and large $\omega$ combinations, there the computational mode carries significant proportion of the initial condition that is lost after a few time-steps only due to large attenuation of the computational mode. Thus, for true unsteady problems where high frequency events are important, the AB-scheme will suppress these events. This is usually the case for all DNS and it is important to note that in [15] simulation of channel flow was performed using this combination of spatial and temporal discretization.

If one replaces $C D_{2}$ by the $C D_{4}$ scheme, one obtains amplification factors from Eq. (22) with $L=\frac{1}{3}[4-\cos (k \Delta x)] \sin k \Delta x$. The other quantities for the $C D_{4}$ scheme are as given in Eqs. (23)-(26) with the changed value of $L$.

In Fig. 6(a) and (b) the time dependent parts of the physical and computational modes, $F$ and $H$-contours are plotted in the $(k \Delta x-\omega \Delta t)$ plane. In Fig. 6(c) and (d) the contours of spectral weights of the initial condition, $M$ and $N$, are shown. The results and the associated problems are qualitatively similar to that for $C D_{2}$ scheme and this combination also cannot be used for DNS.

When the third-order upwind scheme (Eq. (19)) is used for spatial discretization along with AB-scheme, the amplification factors are obtained as roots of the following quadratic equation:

$$
G-1+i \frac{N_{c}}{2}\left(3-\frac{1}{G}\left(L_{2}+i L_{1}\right)\right)=0
$$

And these roots are

$$
\lambda_{1}=F^{\prime} e^{i \eta^{\prime}},
$$$$
\lambda_{2}=H^{\prime} e^{i \Gamma^{\prime}},
$$ 
(a)

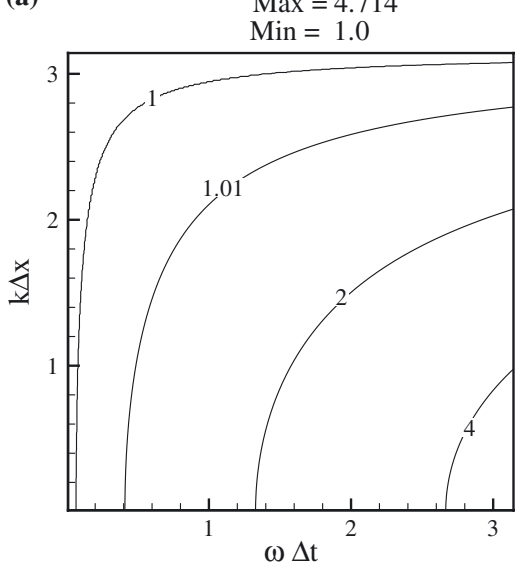

(c)

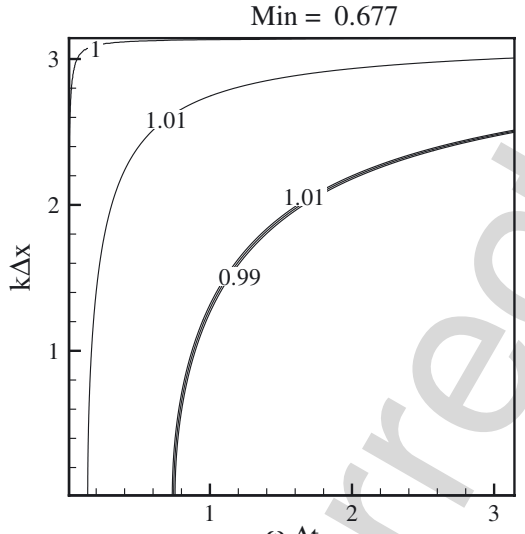

(b)

$\operatorname{Max}=0.335$

$\operatorname{Min}=0.0$

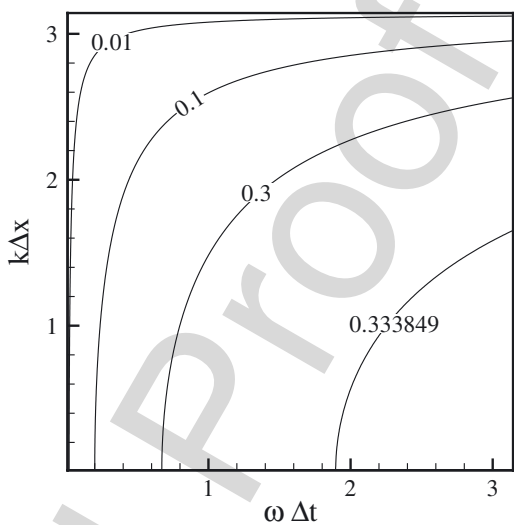

(d)

$\operatorname{Max}=0.323$

$\operatorname{Min}=-0.129$

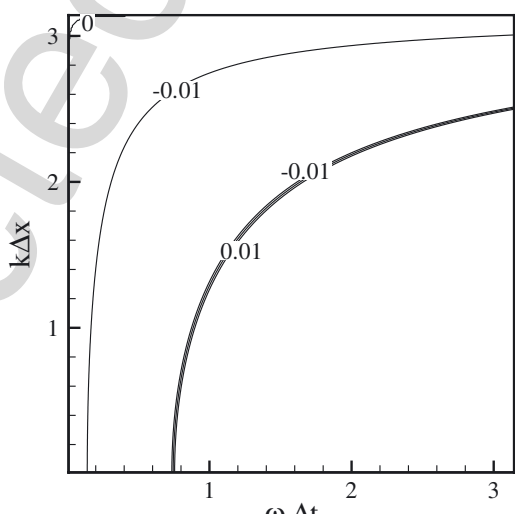

Fig. 5. Amplification factor for solving $1 \mathrm{D}$ wave equation with $\mathrm{AB}$ time- integration and $C D_{2}$ spatial discretization schemes. Time dependent functions: (a) $\mathrm{F}$ for physical; (b) $\mathrm{H}$ for computational modes. Spectral weights of initial condition: (c) M for physical and (d) $\mathrm{N}$ for computational modes.

400 where,

$$
L_{2}=2[4-\cos (k \Delta x)] \sin (k \Delta x),
$$


(a)

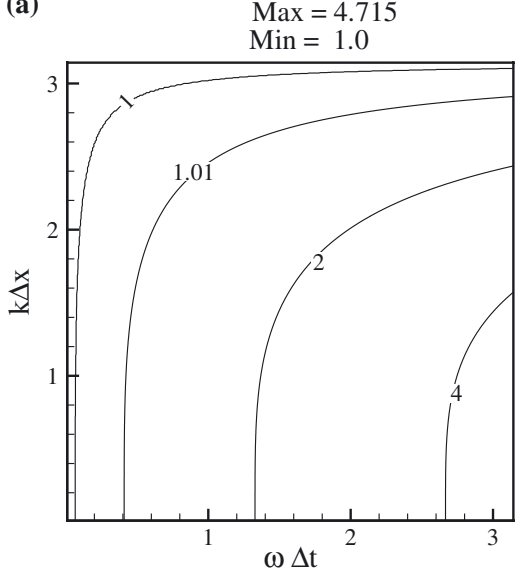

(c)

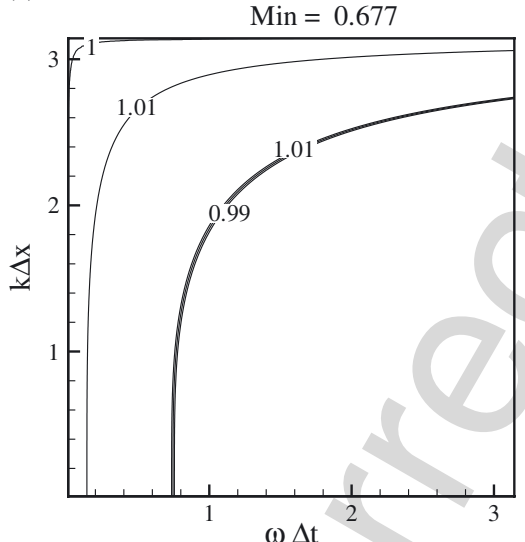

(b)

$\operatorname{Max}=0.335$

$\operatorname{Min}=0.0$

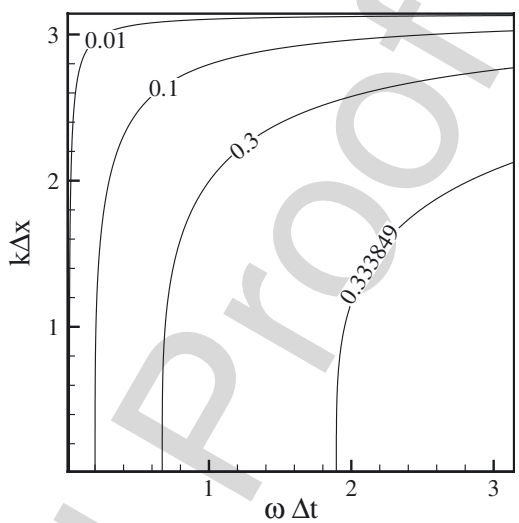

(d)

$\operatorname{Max}=0.323$

Min $=-0.129$

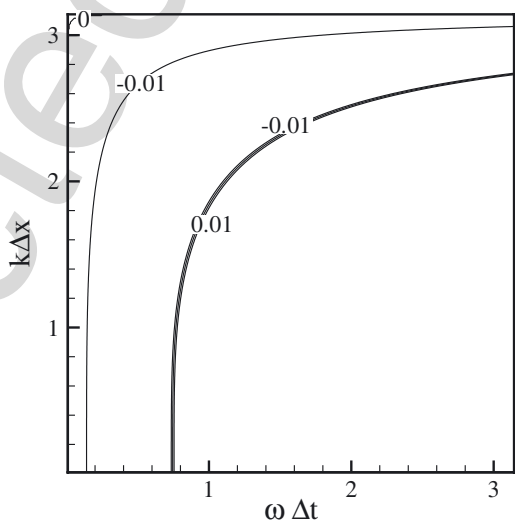

Fig. 6. Amplification factor for solving $1 \mathrm{D}$ wave equation with $\mathrm{AB}$ time- integration and $C D_{4}$ spatial discretization schemes. Time dependent functions: (a) $\mathrm{F}$ for physical; (b) $\mathrm{H}$ for computational modes. Spectral weight of initial condition: (c) M for physical and (d) $\mathrm{N}$ for computational modes.

$$
C^{\prime}=\frac{1}{2}\left[1+\frac{1}{16} N_{c}^{2}\left(L_{1}^{2}+L_{2}^{2}\right)+\frac{N_{c} L_{1}}{2}\right]^{1 / 2},
$$

$$
\tan \left(\bar{\beta}^{\prime}\right)=-\frac{N_{c} L_{2}\left(\frac{1}{3}+\frac{N_{c} L_{1}}{4}\right)}{2+\frac{1}{8} N_{c}^{2}\left(L_{1}^{2}-L_{2}^{2}\right)+\frac{N_{c} L 1}{3}},
$$




$$
\tan \left(\frac{\xi^{\prime}}{2}\right)=-\frac{N_{c} L_{2}}{4+N_{c} L_{1}}
$$

$$
\tan \left(\eta^{\prime}\right)=\frac{C^{\prime} \sin \left(\frac{\xi^{\prime}}{2}\right)+D^{\prime} \sin \left(\frac{\bar{\beta}^{\prime}}{2}\right)}{C^{\prime} \cos \left(\frac{\xi^{\prime}}{2}\right)+D^{\prime} \cos \left(\frac{\bar{\beta}^{\prime}}{2}\right)}
$$

$$
\tan \left(\Gamma^{\prime}\right)=\frac{C^{\prime} \sin \left(\frac{\xi^{\prime}}{2}\right)-D^{\prime} \sin \left(\frac{\bar{\beta}^{\prime}}{2}\right)}{C^{\prime} \cos \left(\frac{\xi^{\prime}}{2}\right)-D^{\prime} \cos \left(\frac{\bar{\beta}^{\prime}}{2}\right)}
$$

The general solution in this case is,

$$
u_{m}{ }^{n}=\int M^{\prime}(k)\left[F^{\prime}\right]^{n} e^{i\left(k x_{m}+n \eta^{\prime}\right)} d k+\int N^{\prime}(k)\left[H^{\prime}\right]^{n} e^{i\left(k x_{m}+n \Gamma^{\prime}\right)} d k
$$

and $M^{\prime}$ and $N^{\prime}$ are obtained from the initial conditions as

$$
\begin{aligned}
& M^{\prime}(k)=A_{0} \frac{\left(1+\frac{N_{c} L_{1}}{6}\right)-H^{\prime} e^{i \Gamma^{\prime}-\frac{i N_{c} L_{2}}{6}}}{F^{\prime} e^{i \eta^{\prime}}-H^{\prime} e^{i \Gamma^{\prime}}} \\
& N^{\prime}(k)=A_{0} \frac{-\left(1+\frac{N_{c} L_{1}}{6}\right)+F^{\prime} e^{i \eta^{\prime}}+\frac{i N_{c} L_{2}}{6}}{F^{\prime} e^{i \eta^{\prime}}-H^{\prime} e^{i \Gamma^{\prime}}} .
\end{aligned}
$$

In Fig. 7(a) and (b) $F^{\prime}$ and $H^{\prime}$ - contours are plotted in the $(k \Delta x-$ $\omega \Delta t)$ - plane. Compared to central schemes, here the computational mode is not negligible for any combination of $k \Delta x$ and $\omega \Delta t$. Furthermore, there are large ranges of $k \Delta x$ and $\omega \Delta t$ for which the computational mode is unstable $\left(H^{\prime}>1\right)$. For physical mode there is very limited ranges of $k \Delta x$ and $\omega \Delta t$ available over which this mode is near-neutral. The physical mode shows instability for practically the whole range of $\omega \Delta t$ when $k \Delta x$ approaches zero. Thus, this scheme has a tendency of instability at the largest length scale for any frequency. Also, this feature of third order upwind scheme explains as to why this produces unstable results as the grid is refined.

The spectral weights, $M^{\prime}$ and $N^{\prime}$ for the initial condition, are plotted in Fig. 7(c) and (d), respectively. One notices that the computational 430 mode significantly contributes to the solution. Also there are ranges of $431 k \Delta x$ and $\omega \Delta t$ over which the computational mode has negative sign. Over432 all, the solution will be contaminated significantly by the computational mode when AB-scheme is used with third order upwind scheme. Next, we write down the various expressions, when OUCS4 scheme is used for spatial discretization along with the $\mathrm{AB}$-scheme. The amplification factors are given by the roots of Eq. (27) where, 
(a)

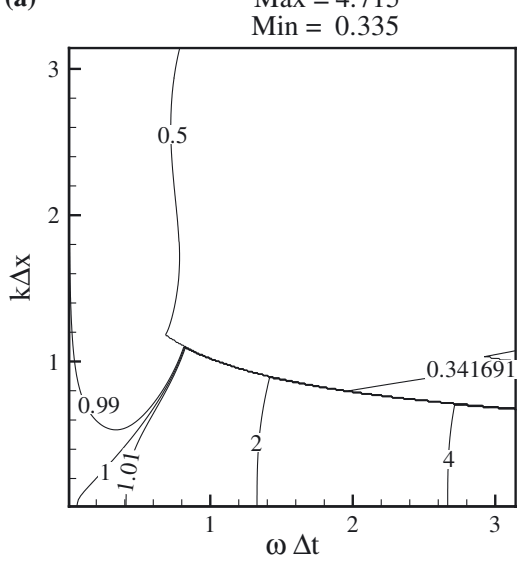

(c)

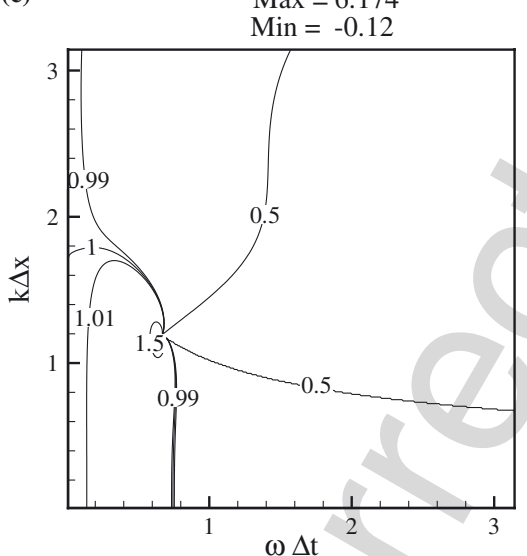

(b)
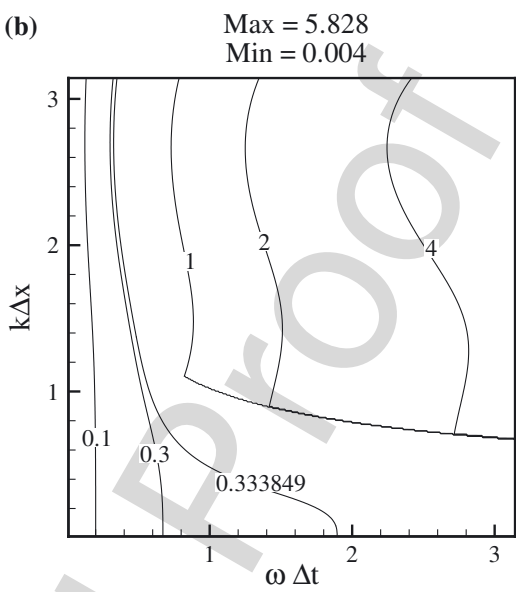

(d)

$\operatorname{Max}=1.123$

Min $=-5.174$

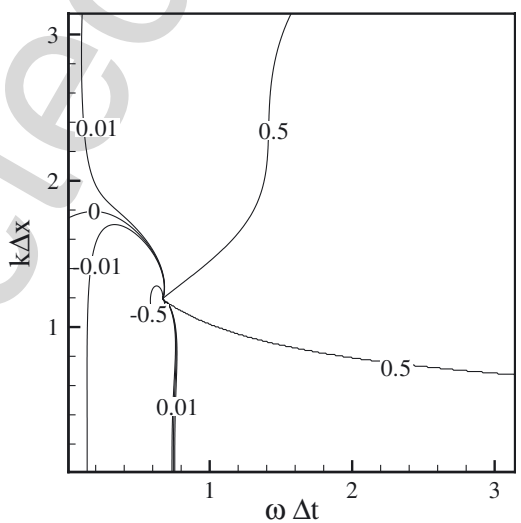

Fig. 7. Amplification factor for solving $1 \mathrm{D}$ wave equation with $\mathrm{AB}$ time- integration and $U D_{3}$ spatial discretization schemes. Time dependent functions: (a) F' for physical; (b) $\mathrm{H}^{\prime}$ for computational modes. Spectral weight of initial condition: (c) M' for physical and (d) N' for computational modes.

$$
L_{1}=-6 \sum_{l=1}^{N} C_{j l} \cos ((l-j) k \Delta x) \text {, }
$$

$$
L_{2}=6 \sum_{l=1}^{N} C_{j l} \sin ((l-j) k \Delta x) .
$$


438 The general solution in this case is given as in Eq. (30). The time dependent and the independent parts are as given in Eqs. (29) and (31).

If we replace the OUCS4 by SS scheme, then the amplification fac441 tors are obtained from Eq. (22) with $L=a_{0} \sin (k \Delta x)+\frac{b_{0}}{2} \sin (2 k \Delta x)+$ $442 \frac{d_{0}}{3} \sin (3 k \Delta x)+\frac{e_{0}}{4} \sin (4 k \Delta x) . a_{0}, b_{0}, d_{0}$, and $e_{0}$ are the same as in Eq. (12). 443 The general solution is as given in Eq. (25) and the numerical phase speed, group velocity etc. are calculated from Eqs. (24) and (26).

In Fig. 8(a) and (b) the time dependent parts, $F^{\prime}$ and $H^{\prime}$, are plotted for the OUCS4 scheme used with the AB-scheme. It is seen that OUCS4 scheme will perform well only when $\omega \Delta t$ is restricted to a small valuebeyond which the physical mode is unstable. It is noted that the computational mode is unstable for large $k \Delta x$ and $\omega \Delta t$ combinations. Overall, this scheme will work for small time steps. Fig. 8(c) and (d) show the variation of time independent parts of the general solution and this shows that the computational mode has less contribution as compared to the other schemes discussed before.

In Fig. 9(a)-(d) the corresponding information is given for the SS scheme. The behavior of this scheme is similar to other central schemes discussed before. The computational mode will be important for DNS when large $\omega \Delta t$ values are present.

The scaled numerical phase speed contours are shown in Fig. 10 for the physical and computational modes for $C D_{2}, C D_{4}$ and the third order upwind schemes. The physical mode shows desirable property on a small patch near the origin for all the schemes. The computational mode has very high phase speed for all length scales and very small $\omega \Delta t$ for all the schemes. For the third order scheme there is a line, across which phase of the computational mode display discontinuous jump.

In Fig. 11(a), (c), and (e) the numerical phase speed contours of OUCS4, SS scheme for interior points and SS scheme for the second point are shown for the physical mode. All the three figures show a large range of $k \Delta x$ over which the numerical phase speed is close to the actual value for small $\omega \Delta t$ - the range for $k \Delta x$ is twice the value obtained for the schemes shown in Fig. 10. The contours for the numerical phase speed for the computational mode of these schemes are shown in Fig. 11(b), (d), and (f). These show very large phase speeds for small $\omega \Delta t$ values.

In Fig. 12 and 13 the numerical group velocity contours are plotted in $(k \Delta x-\omega \Delta t)$ - plane for both the modes for AB-scheme. Figure 12 shows the scaled numerical group velocity components for the physical and computational modes for $C D_{2}, C D_{4}$ and the third order upwind schemes of spatial discretization. The corresponding results are shown for OUCS4 and SS spatial discretization schemes in Fig. 13. The variations are quali- 
(a)

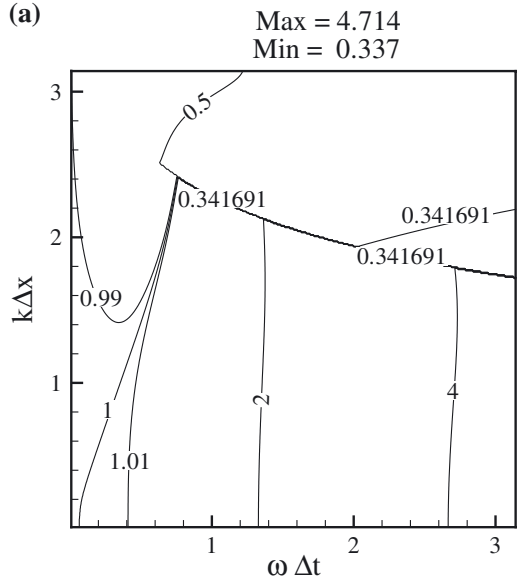

(c)

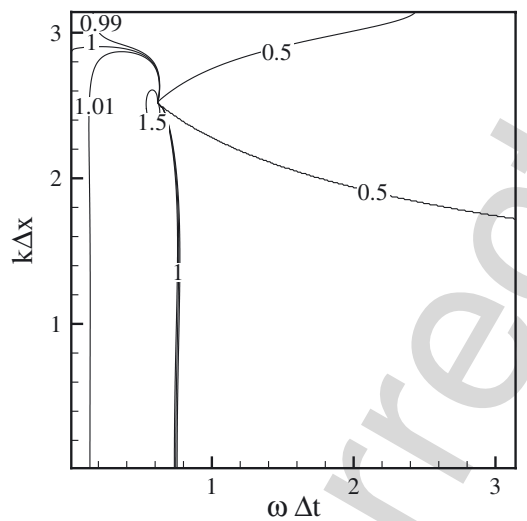

(b)

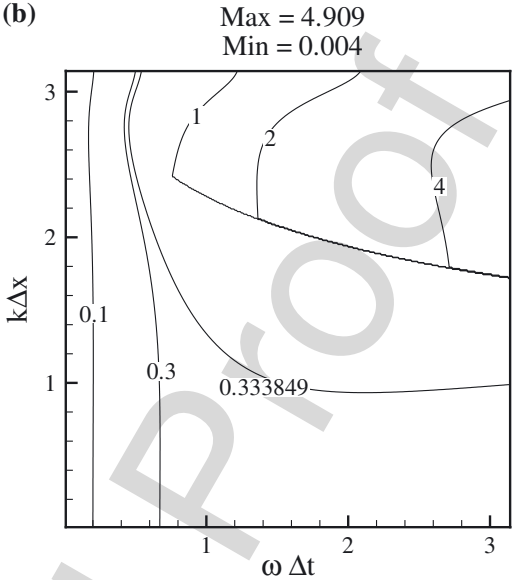

(d)

$\operatorname{Max}=1.048$

Min $=-4.598$

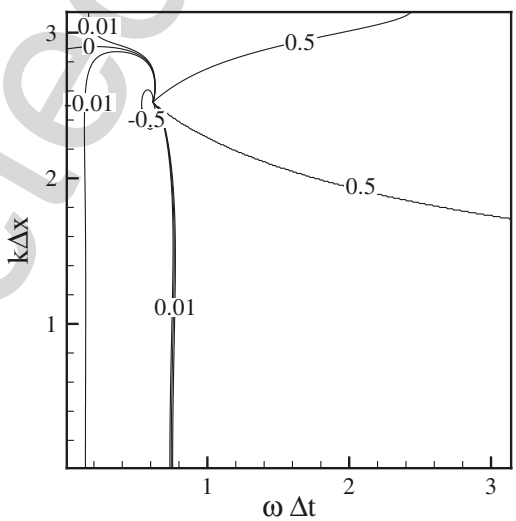

Fig. 8. Amplification factor for solving $1 \mathrm{D}$ wave equation with $\mathrm{AB}$ time- integration and OUCS4 spatial discretization scheme. Time dependent functions: (a) F' for physical; (b) H' for computational modes. Spectral weight of initial condition: (c) M' for physical and (d) N' for computational modes.

tatively the same for the physical mode as the numerical phase speed variations shown in Fig. 11 for OUCS4 and SS schemes. Both the physical and computational modes for the $C D_{2}$ and $C D_{4}$ schemes show a straught line along which the group velocity is zero. For the third order scheme the zero group velocity line is curved. For the $C D_{2}$ and $C D_{4}$ schemes, this 484 line also shows an interesting feature. If the physical mode travels from left to right, the corresponding computational mode travels from right to left 
(a)

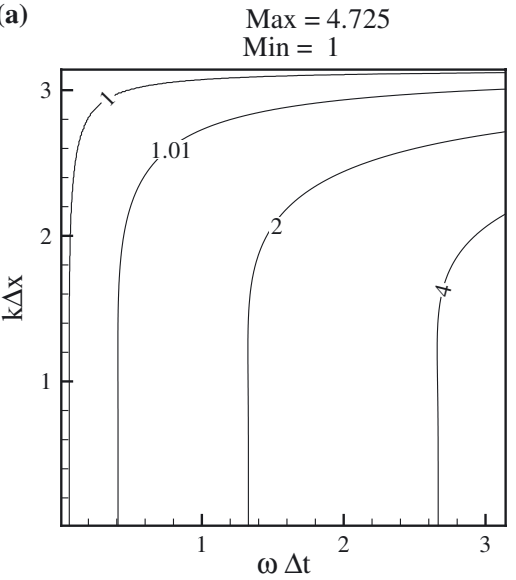

(c)

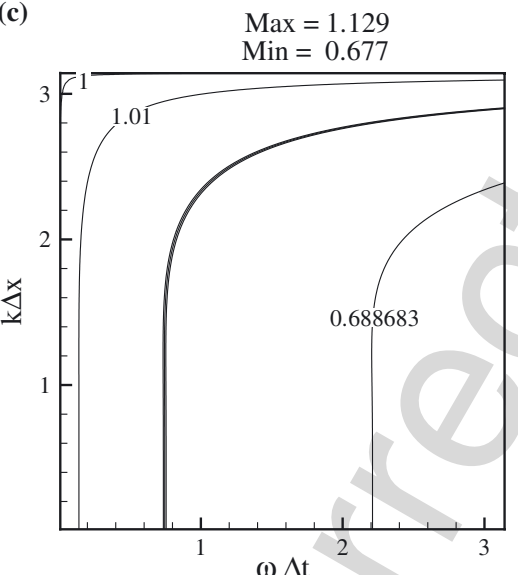

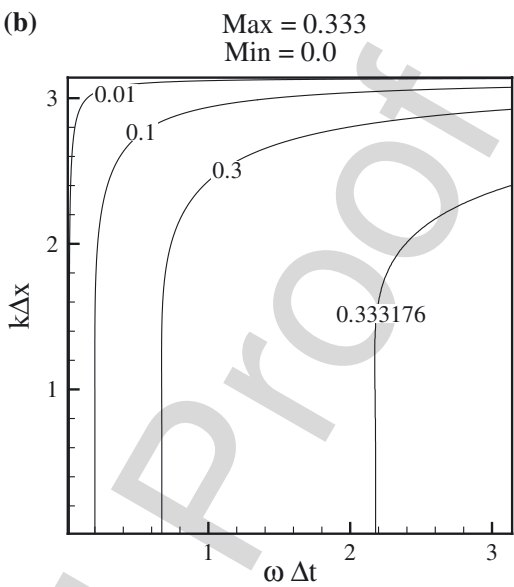

(d)

$\operatorname{Max}=0.323$

Min $=-0.129$

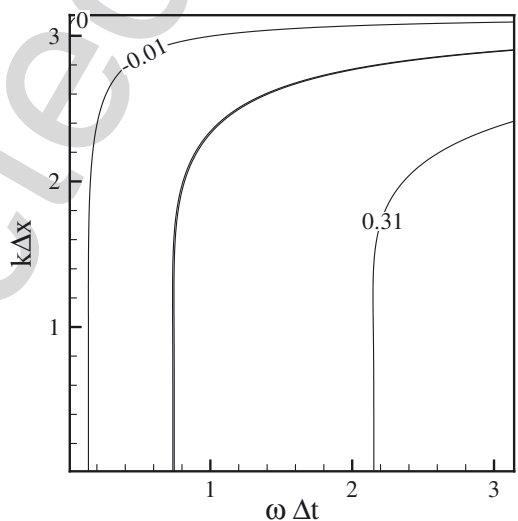

Fig. 9. Amplification factor for solving $1 \mathrm{D}$ wave equation with $\mathrm{AB}$ time- integration and SS spatial discretization scheme. Time dependent functions: (a) $\mathrm{F}$ for physical; (b) $\mathrm{H}$ for computational modes. Spectral weight of initial condition: (c) M for physical and (d) $\mathrm{N}$ for computational modes.

and vice versa. Similar features also holds good for the third order upwind scheme. As compared to the schemes of Fig. 12, OUCS4 and SS schemes have better DRP property, as shown in Fig. 13. However, the computational mode has wider variations and the SS interior scheme does not dis-

490 play any upstream propagating mode. 

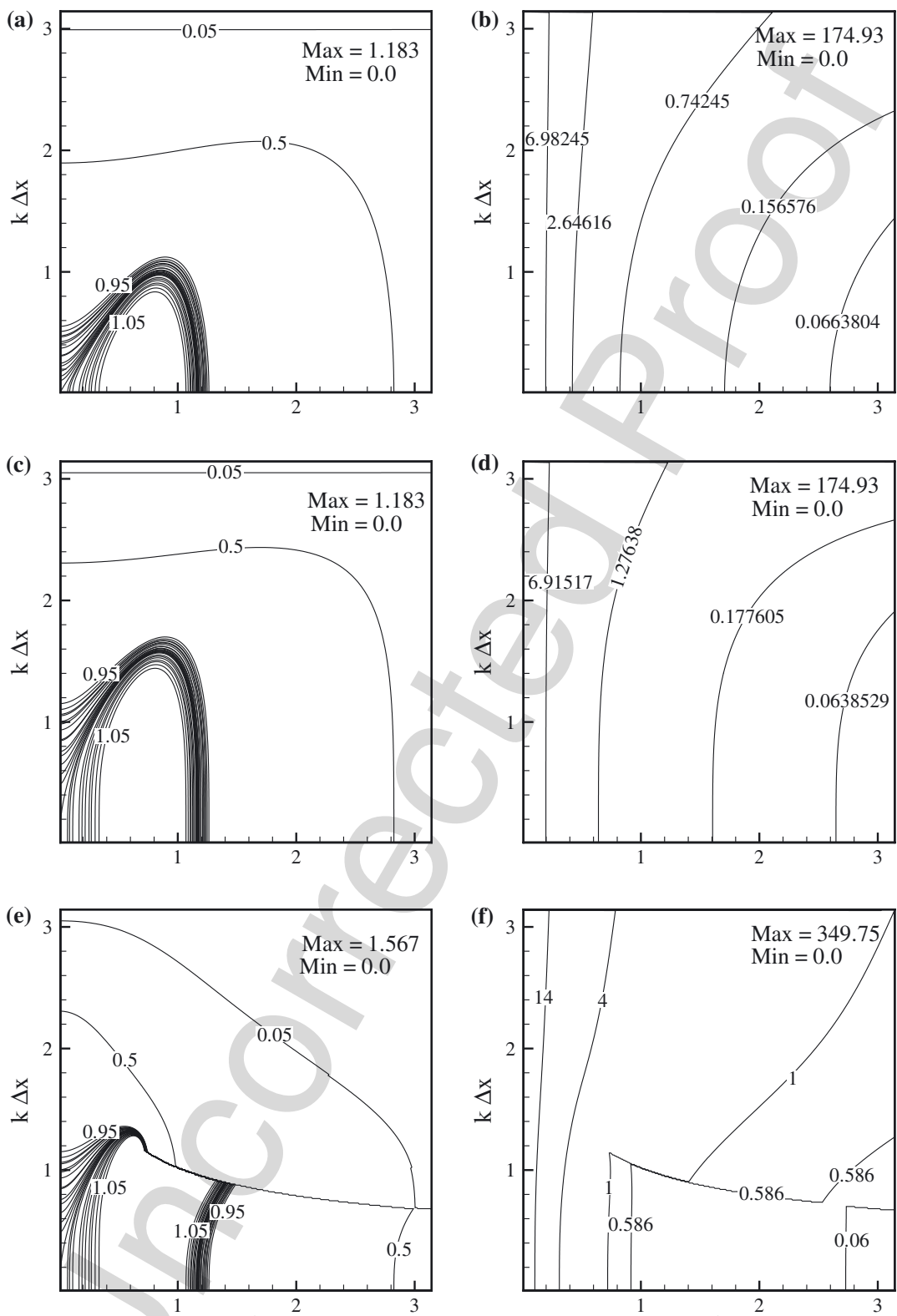

$\omega \Delta \mathrm{t}$

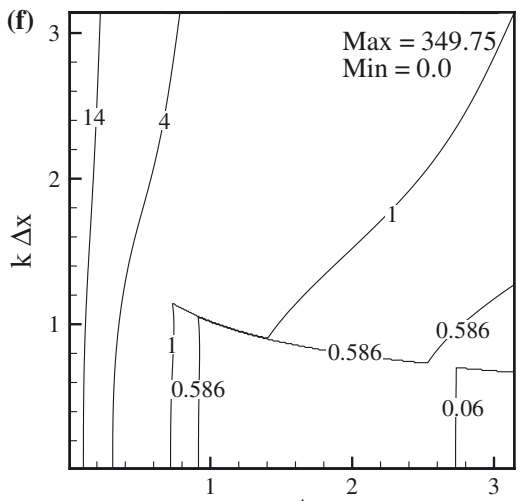

$\omega \Delta \mathrm{t}$

Fig. 10. Scaled numerical phase speed $\left(c_{N} / c\right)$ for $1 \mathrm{D}$ wave equation with $\mathrm{AB}$ time-integration scheme. Figure (a), (c) and (e) show physical mode of $C D_{2}, C D_{4}, U D_{3}$; (b), (d) and (f) show computational mode of $C D_{2}, C D_{4}$ and $U D_{3}$, respectively. 

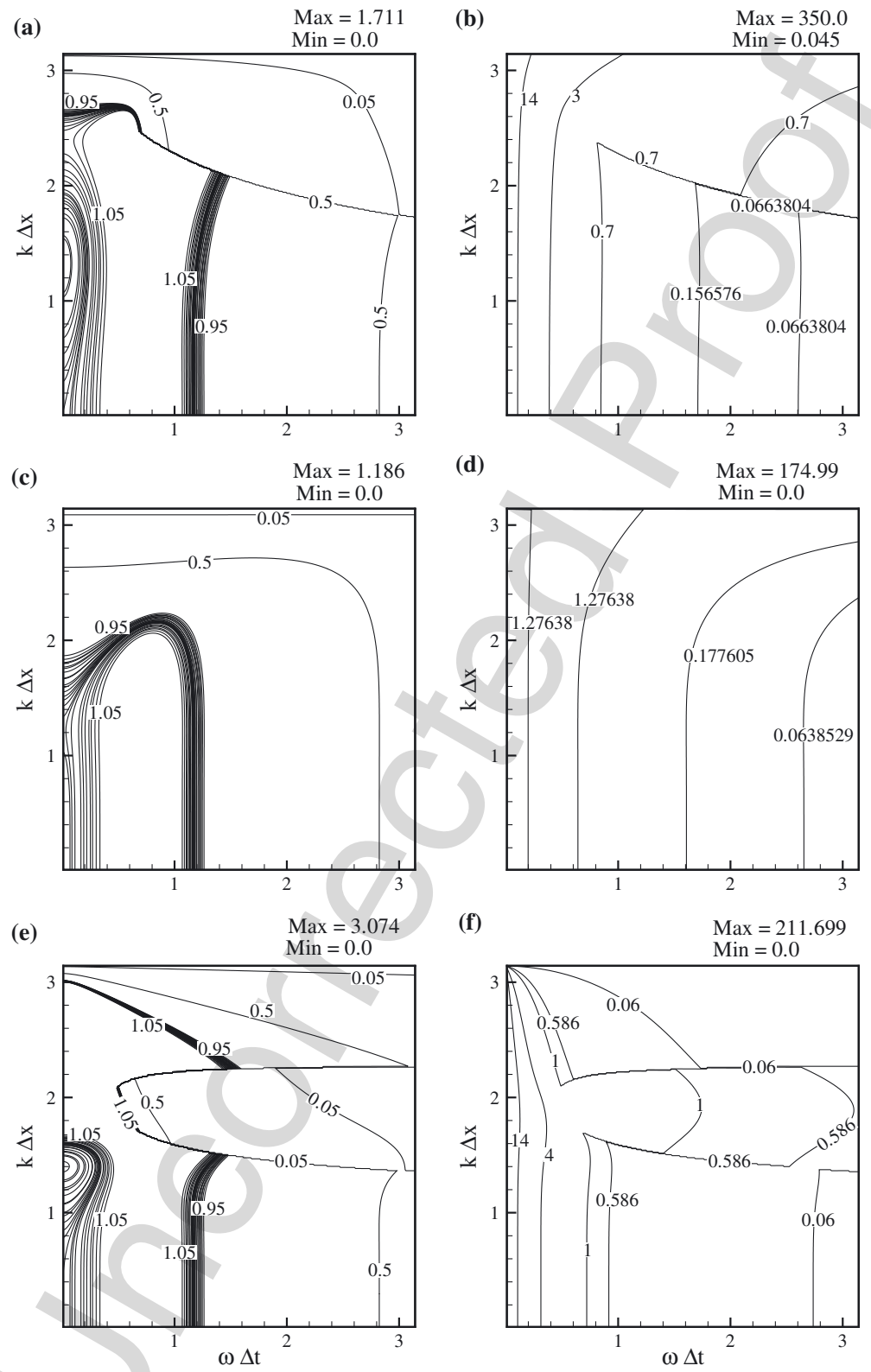

Fig. 11. Scaled numerical phase speed $\left(c_{N} / c\right)$ for $1 \mathrm{D}$ wave equation with $\mathrm{AB}$ time-integration scheme. Figure (a), (c), (e) show physical mode and (b), (d), (f) show computational mode of OUCS4, SS-interior and SS-second scheme, respectively. 

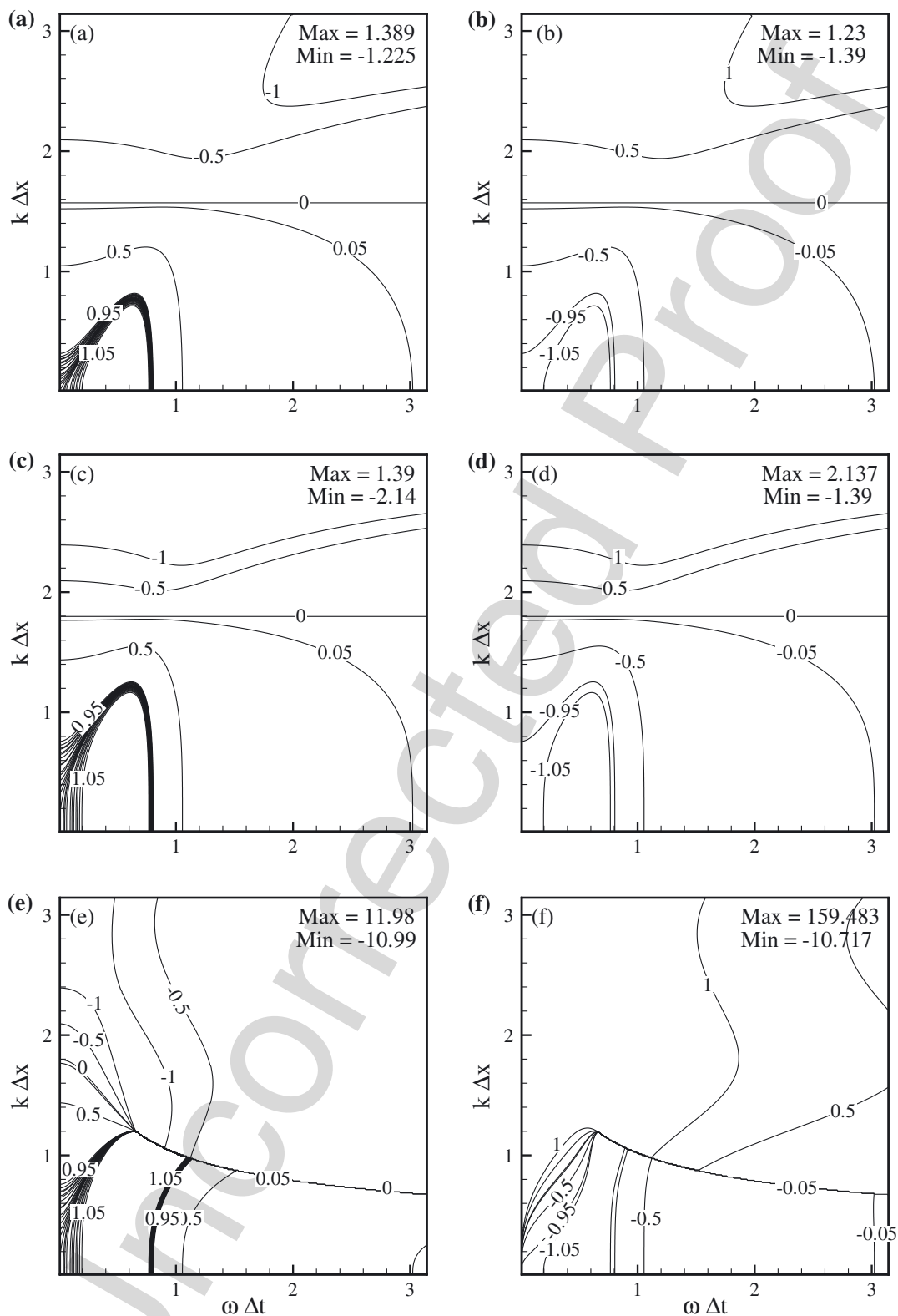

Fig. 12. Scaled numerical group velocity $\left(V_{g N} / c\right)$ for $1 \mathrm{D}$ wave equation with $\mathrm{AB}$ time-integration scheme. Figure (a), (c) and (e) show physical mode and (b), (d) and (f) show computational mode of $C D_{2}, C D_{4}$ and $U D_{3}$ scheme, respectively. 
(a)

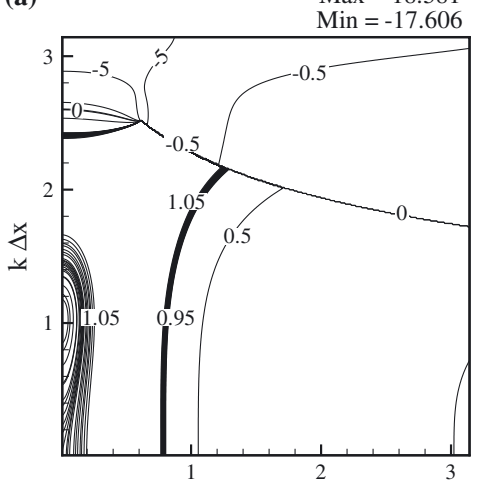

(c)

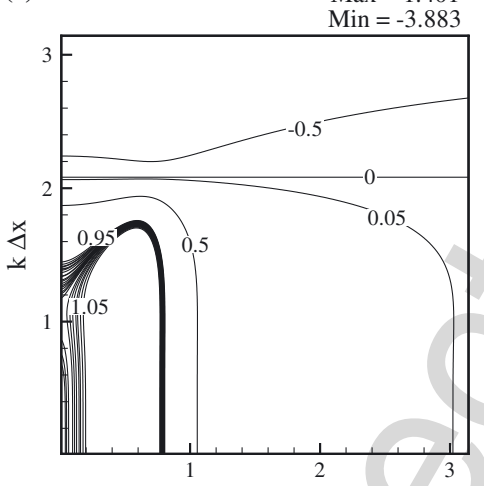

(e)

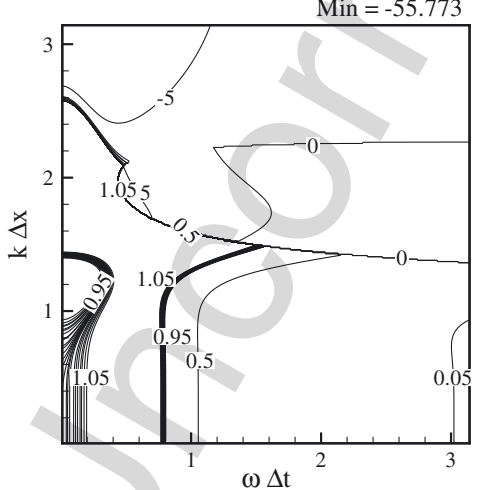

(b)

Max $=1340.45$

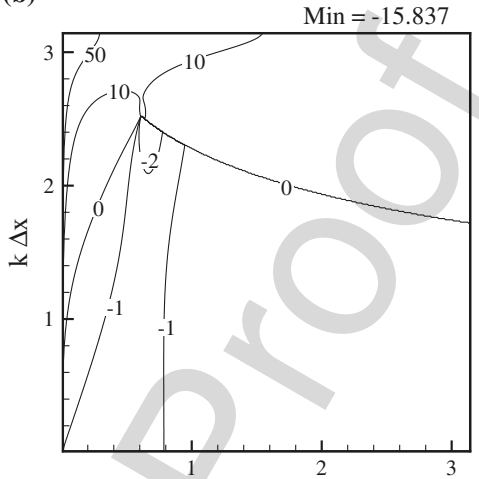

(d)
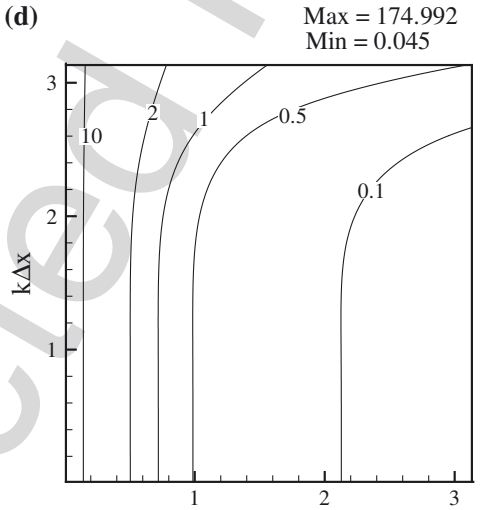

(f)

Max $=128.088$ Min $=--690.383$

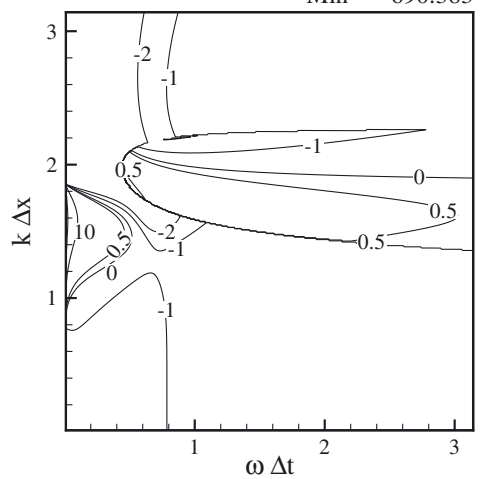

Fig. 13. Scaled numerical group velocity $\left(V_{g N} / c\right)$ for $1 \mathrm{D}$ wave equation with $\mathrm{AB}$ time-integration scheme. Figure (a), (c), (e) show physical mode and (b), (d), (f) show computational mode of OUCS4, SS-interior and SS-second scheme, respectively. 


\subsection{Four Stage Runge Kutta Method}

Following the same methodology discussed in the previous two subsections and using the symbolic toolbox of MATLAB we have estimated the amplification rate, scaled numerical phase speed and numerical group velocity for $C D_{2}$ and $C D_{4}$ schemes when used with RK4 time integration scheme. The results are shown in Fig. 14. For both the spatial schemes, the amplification factor displays a large range of $\omega \Delta t$ over which the scheme is neutrally stable- a very desirable feature of DNS methodology. While the range of wave numbers and frequencies over which this is true is identical for both the schemes, it is the numerical phase speed and group velocity that shows difference between these two methods. In both respects, $C D_{4}$ scheme performs better than $C D_{2}$ scheme. The range of $k \Delta x$ can be further increased over which DRP property is maintained, if one replaces the $C D_{4}$ scheme by compact schemes. Such a scheme is used for the acoustics problem discussed in the following section.

\section{6}

\section{ILLUSTRATIVE EXAMPLES}

Here we demonstrate some of the properties of the schemes discussed in the previous two sections. We choose two problems to highlight the problems of spurious computational mode in using multilevel time integration schemes.

\subsection{Solving Navier-Stokes Equation Using Third Order Upwind Scheme}

The results of Sec. 2 clearly reveals that for high Reynolds number flows central schemes are unsuitable when used with either Euler or ABscheme due to numerical instability. This is avoided by switching over to upwind schemes those having a range of $k \Delta x$ for which the schemes are stable when used with Euler time integration scheme. To avoid changing physical dissipation while stabilizing computations, it is practical to use third order upwind schemes, as the one given by Eq. (19). Considering numerical instability one is restricted to very small time steps when third order upwind scheme is used. In contrast, the physical mode of AB-scheme allows taking much larger time steps. But the major problems arise, as the computational mode is non-negligible and has non-physical contributions including a part of the energy at large length scales that propagates upstream - as indicated by the group velocity.

The above observations are demonstrated here by solving NavierStokes equation for flow past a rotating circular cylinder, using the third order upwind scheme for spatial discretization and Euler and $\mathrm{AB}$ - scheme 

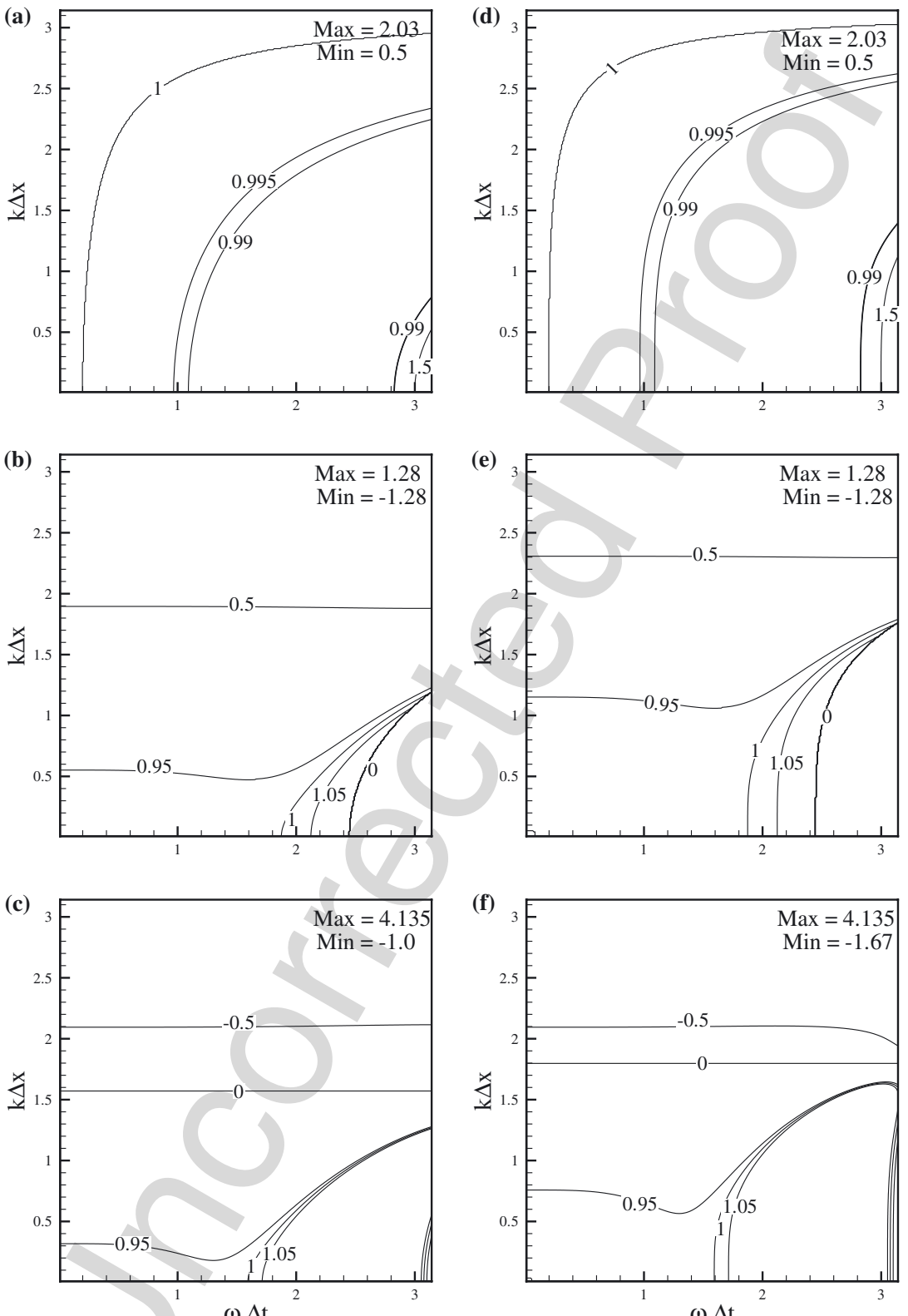

Fig. 14. Amplification factor (a) and (d), scaled numerical phase speed (b) and (e) and scaled numerical group velocity (c) and (f) contours for RK4 time -integration scheme with: (a)-(c) $C D_{2}$ and (d)-(f) $C D_{4}$ scheme. 
528 for temporal discretization. The physical problem is chosen for the uni529 form flow at $R e=3800$ and a non-dimensional rotation rate, $\Omega=10$ i.e. 530 the peripheral speed of the cylinder is ten times the free-stream speed. 531 Flow past rotating cylinder for this type of flow parameters display phys532 ical instabilities- as reported in [25]. In a recent work [26] a possi-

533 ble explanation for the temporal instabilities is provided. In [26], the 534 Navier-Stokes equation is solved using $(\psi-\omega)$ formulation that uses third order upwind scheme with Euler time integration scheme for $\Omega=5$. It was noted that the flow suffered temporal instabilities after an impulsive start-up. During these instabilities the loads change abruptly at discrete times.

539 Here the results are compared between Euler and AB-time integra540 tion strategies using the same methods but at the higher rotation rate of $541 \Omega=10$. In solving this problem a fine grid with 450 points in the radial 542 direction and 271 points in the azimuthal direction have been taken. The 543 first azimuthal line is $0.0005 \mathrm{D}$ distance away from the cylinder and the outer boundary is located $24 \mathrm{D}$ from the cylinder. A non-dimensional time step of 0.0001 have been used for both the time integration strategies.

546 The lift and drag coefficients are shown in Fig. 15, where Euler and AB547 schemes are used to advance the vorticity transport equation. For this 548 high rotation rate case, Euler time integration once again displays tem549 poral instabilities at discrete times. This instability was shown in [26] to 550 arise from a mechanism where a given equilibrium flow is destabilized by 551 far-field disturbance and as a consequence, lump of vorticity that is con552 fined within the recirculating fluid around the cylinder is released in the 553 wake of the cylinder. However, when the AB-scheme is used, the compu554 tational mode, has negative group velocity for combinations of small values of $k \Delta x$ and $\omega \Delta t$. As these are useful excited length and time scales the computational mode prevents the lump of vortex to be released in the wake. As a consequence the instabilities are weakened and in the $C_{l}$ and $C_{d}$ vs time plots the discrete jumps in the value are smoothed out. This is a demonstration of the spurious behavior of $\mathrm{AB}$-scheme in solving time dependent problems where a large range of length and time scales are excited.

\subsection{Solving Euler Equation For A Fluid Medium Excited by Pulses}

The effectiveness of the schemes discussed in Sects. 2 and 3 will be attempted here with the standard example that was introduced in [9], where three Gaussian pulses are introduced in an uniform flow $\left(M_{\infty}=0.5\right)$ and the response of the system is numerically calculated and compared with the exact solution. At $t=0$, a pressure pulse is taken at the center of 

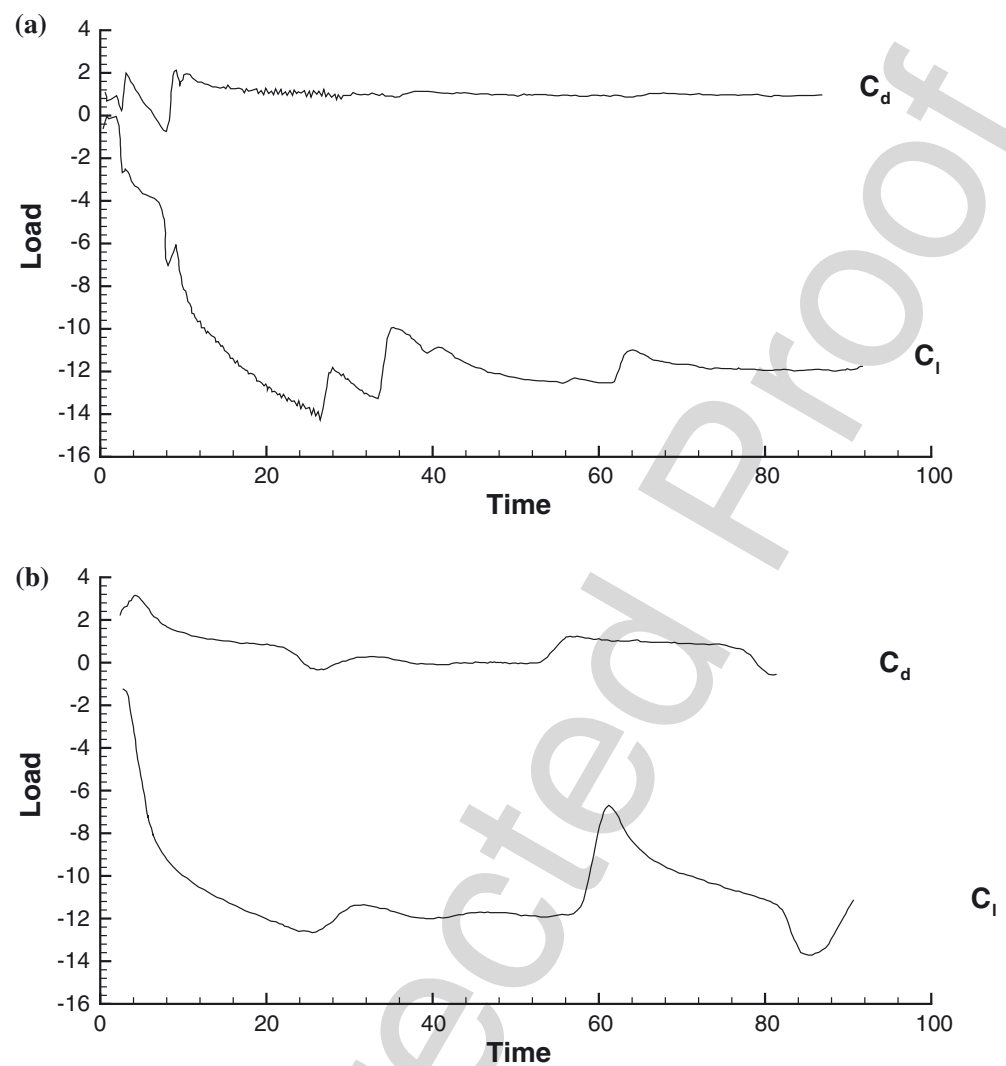

Fig. 15. The calculated lift and drag coefficients for $\operatorname{Re}=3800$ and $\Omega=10.0$ as a function of time for impulsive start case using (a) Euler and (b) AB time- integration scheme.

568 the domain along with a vorticity and an entropy pulse taken downstream 569 of the pressure pulse at a distance equal to $1 / 3$ of the length of the com570 putational domain. All these pulses reach the outflow boundary simulta571 neously. We take the same computational parameters and amplitude and 572 half width of the Gaussian pulses, as were taken in [9]. The codes are 573 written for the full Euler equation and the disturbance solution can be 574 extracted from it to compare with the exact solution.

$$
\frac{\partial U}{\partial t}+\frac{\partial E}{\partial x}+\frac{\partial F}{\partial y}=H,
$$

$576 \quad$ where $U=|\rho \rho u \rho v \rho e|^{T}$

$577 E=\left|\rho u \rho u^{2}+p \rho u v \rho u h\right|^{T}$ and $F=\left|\rho v \rho u v \rho v^{2}+p \rho v h\right|^{T}$ 
$578 H$ represents the forcing term that is zero in the present case. The fol579 lowing initial conditions for different primitive variables, instead, drive the 580 fluid dynamical system: where $r^{2}=x^{2}+y^{2}$ and the quantities with subscript $d$ represent disturbance components. We have used the same scales as those used in [9] and $\epsilon_{1}=0.01, \epsilon_{2}=0.1$ and $\epsilon_{3}=0.0004$ are the amplitudes of the pressure, entropy and the vorticity Gaussian pulses respectively. The half- width of the respective pulses $\left(b_{i}\right)$ are 3,5 and 5-the same that was used in [9]. This defines $\alpha_{i}=\frac{\ln 2}{b^{2}}$.

In solving Eq. (33) with the initial condition given by Eq. (34), we used the same radiation and outflow boundary conditions that are given in [9]. We have solved the full Euler equation (Eq. (33)) using the different spatial schemes and three time integration schemes. Apart from Euler and $\mathrm{AB}$-scheme we have used the RK4 time integration scheme. The RK4 scheme was specifically chosen because this is a higher-order scheme, but it does not have any spurious computational mode. We have solved the equation in the physical plane using $(200 \times 200)$ uniform grid and a CFL number of 0.5 and this fixes $\Delta t=0.0569$. For all the schemes we used different layers of ghost cells on all four segments of the boundary to check the effectiveness of outflow and radiation boundary conditions. In [9] three layers of ghost cells were used, because their spatial discretization scheme used seven point explicit stencil. In the SS scheme we have developed a nine point stencil that requires four layers of ghost cell. It is to be noted that the usage of four layers of ghost cells in 2D can be a matter of concern for $3 \mathrm{D}$ computations, where a very large numbers of points need to be added. For example, for a grid of size $(M \times N \times K)$, the added number of ghost cells are given by $8[M N+M K+N K+8 M+8 N+8 K+64]$. However, for the compact schemes ghost cells are not required per se, but we have used them to avoid spurious reflections from the boundary segments.

First, we compare the exact solution with the numerical solutions using few combinations of spatial and temporal discretization schemes in Fig. 16. Figure 16(a) and (b) show the comparison of the density and the pressure disturbance of the numerical schemes along with the exact solution at $500 \Delta t$ and $2000 \Delta t$, using four layers of ghost cells. All the schemes 

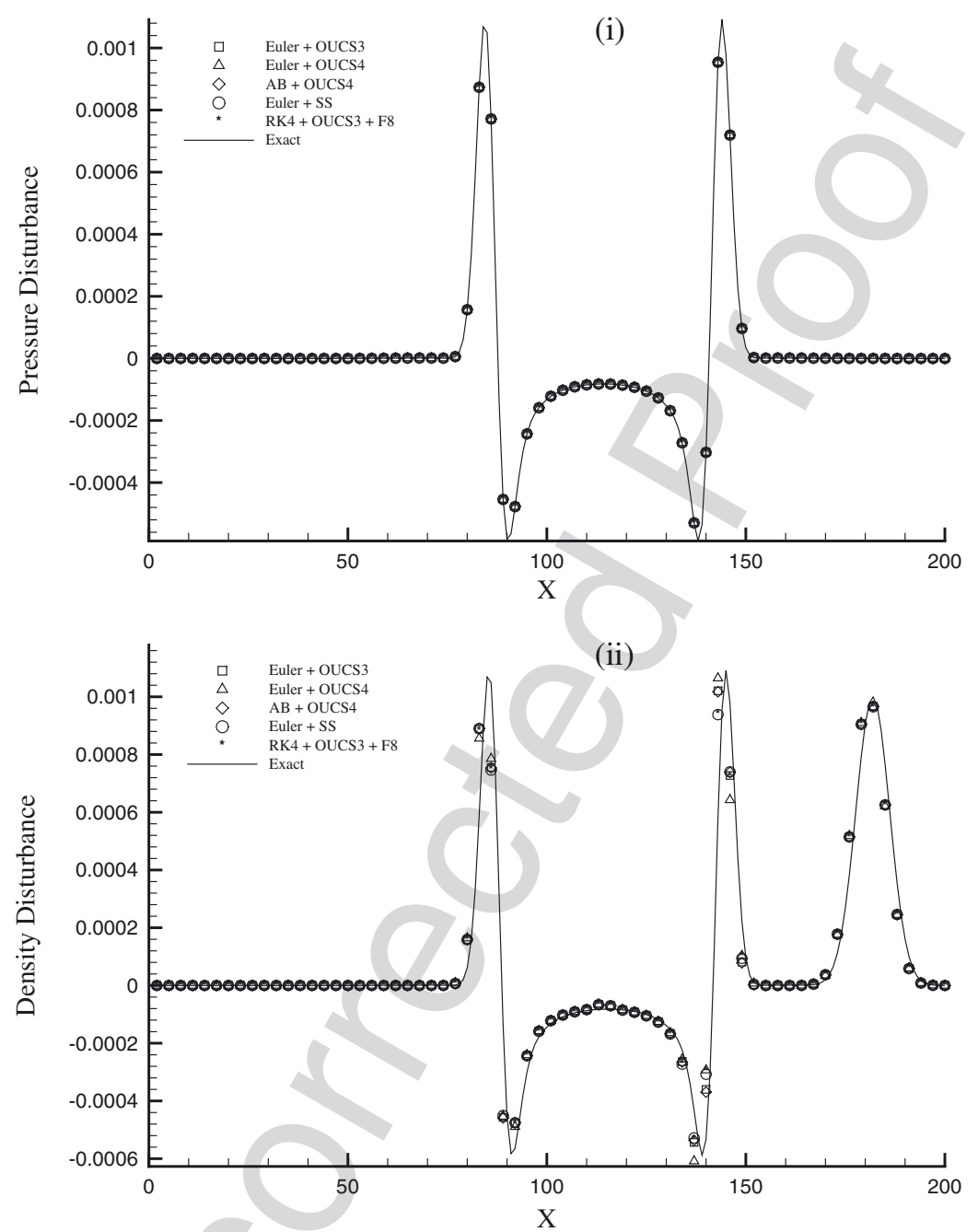

Fig. 16(a). Exact solution of Eqs. (33) and (34) compared with computed solutions after $500 \Delta t$, using the indicated space-time schemes using 9-pt. stencil with four layers of ghost cells. Figure (i) and (ii) show the pressure and density disturbance respectively.

617 match quite well with the exact solution, except the results shown with 618 the AB-scheme at later times, as was stated explicitly in [13] and quoted 619 in the Introduction. The computed pressure waveform along the $x$-axis 620 matches with the exact solution for all times for the other schemes. How621 ever, density contours show a marginal mismatch with the exact solution. 

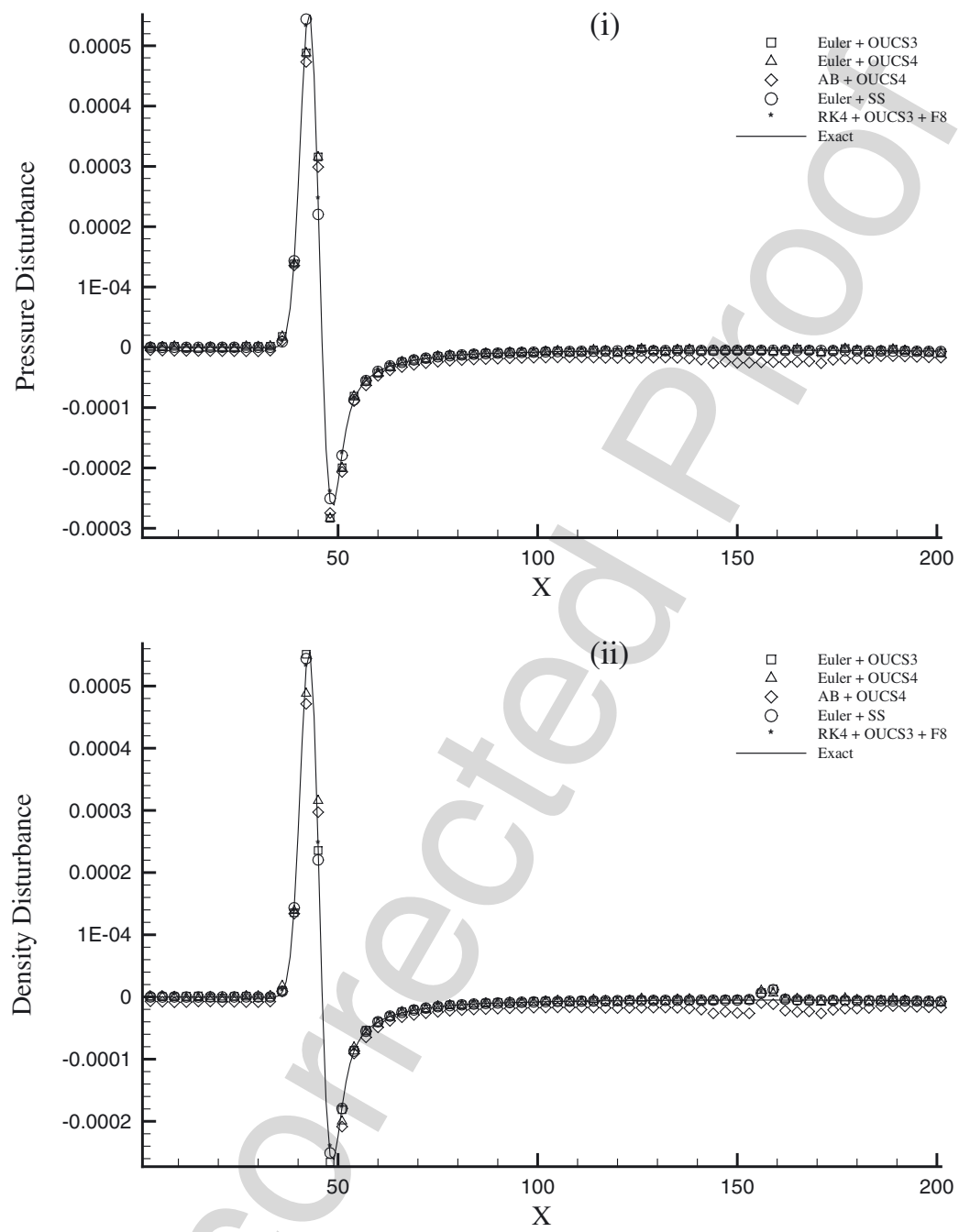

Fig. 16(b). Exact solution of Eqs. (33) and (34) compared with computed solutions after $2000 \Delta t$, using the indicated space-time schemes using 9-pt. stencil with four layers of ghost cells. Figure (i) and (ii) show the pressure and density disturbance respectively.

622 It is interesting to note that there is a small dispersion between the two 623 solutions at $500 \Delta t$, which however disappears at $2000 \Delta t$ and higher times. 624 It is to be noted that the exact solution is in reality the asymptotic solu625 tion that is due to the poles and singularities near the origin in the spec626 tral plane. If there are any higher modes and essential singularities that 
627 are away from the origin in the $\omega$ - plane, then they will be responsible for 628 the transients. A bump in the density contour is noted for $2000 \Delta t$ which 629 is of the order of $1 \%$ of the peak amplitude. This is obtained for all the 630 spatial and temporal discretization combinations and the same bump was 631 also noticed in [9] in Fig. 7. Even when the four layers of ghost cells were 632 used, the RK4 time integration scheme produced spurious reflections from 633 the inflow boundary. To remedy this we used an $8^{\text {th }}$ order Filter $(F 8)$ as 634 given in [8].

635 Having seen that all the schemes show good agreement with the exact 636 solution along the $x$-axis, it is natural to compare the solution at other 637 locations next. This has been attempted by plotting the contours for den638 sity, pressure and speed in the full computational domain in Fig. 17 and 63918

640 In Fig. 17(a) the results are shown for OUCS3 spatial scheme 641 used with Euler time discretization scheme. Similarly for the results in 642 Fig. 17(b) and (c) the same spatial scheme, but RK4 and AB-time dis643 cretization schemes have been used. For each combinations of spatial 644 and temporal discretization schemes, we have used Five, seven and nine 645 point stencils at the boundary for the Euler time integration scheme in 646 Fig. 17(a). All the three quantities show that the five point stencil is inad647 equate to prevent reflections from the boundary. Thus in other time inte648 gration schemes we do not show five point stencil results. If we compare 649 Fig. 17(a) and (b), we find that the density and pressure contours for 650 Euler and RK4 schemes match well, however the speed contours in the 651 Euler time scheme show oscillations. For the AB-time integration scheme 652 the results, as shown in Fig. 17(c), for density and pressure contours are in 653 good agreement with Euler and RK4 schemes, with no oscillations. How654 ever, the central core in density contour disappears as compared to the 655 results of Fig. 17(a) and (b) and also with similar results shown in [9]. 656 The speed contours do not match with the other time integration schemes 657 with gross mismatch in the mean value itself. This is due to the large 658 dissipation associated with the computational mode as discussed in Sec. 2.2. 659 Among the three time integration schemes RK4 performs the best only 660 when an 8th-order filter is used. Euler scheme will however, be preferred if 661 the high frequency small wavelength oscillations, noted in speed contours, 662 are removed by applying a high order filter.

663 For OUCS4 spatial scheme, one notices similar features of the solu664 tion for all the time integration schemes, as was noted for OUCS3 scheme. 665 The AB-time integration scheme shows large errors and even the mean 666 flow is distorted. Thus, this time integration scheme cannot be used for 667 computational acoustics problems. It is to be pointed out that the basic 668 OUCS4 scheme has inherent numerical instability problem at the near 

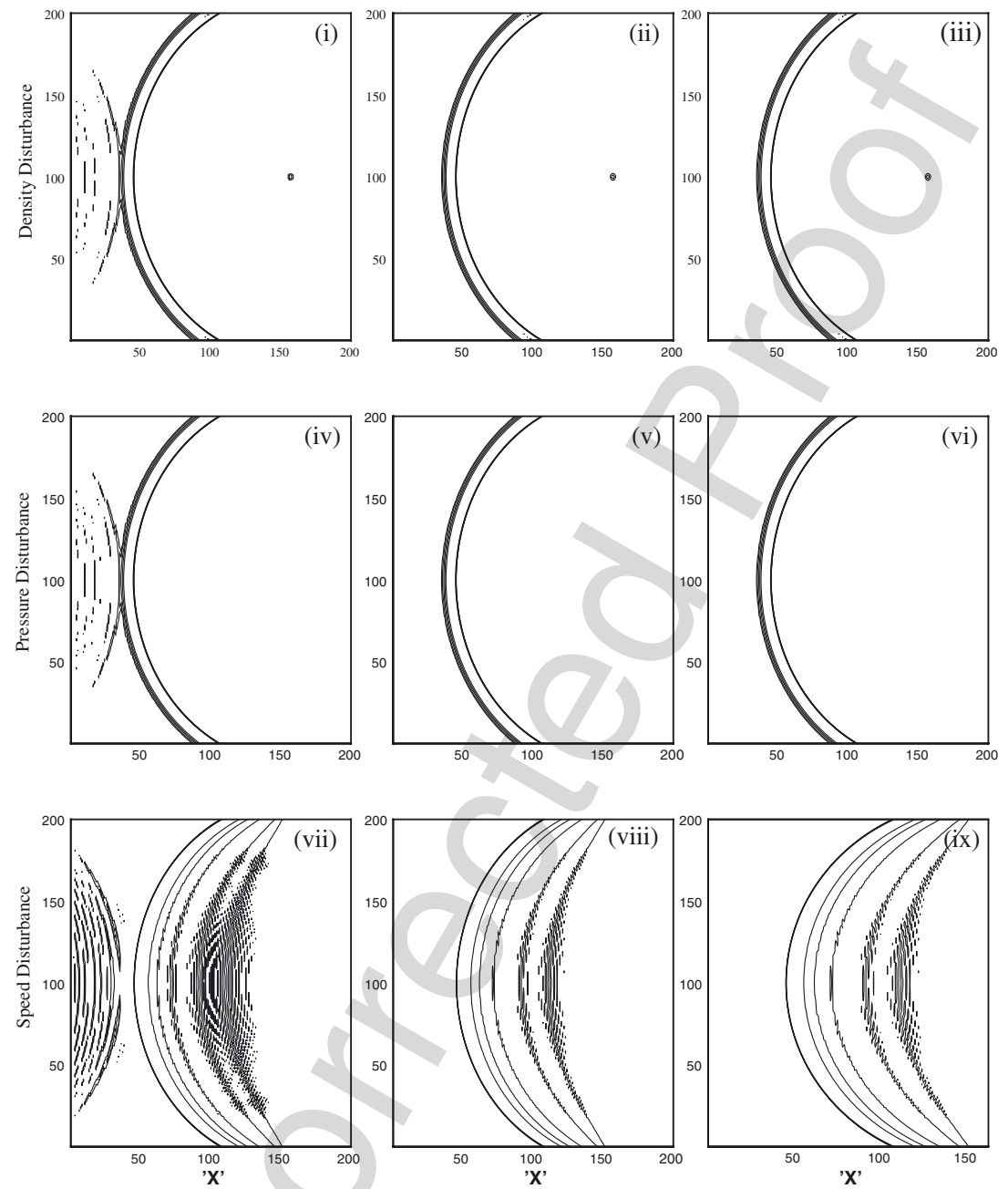

Fig. 17(a). Solution of Eqs. (33) and (34) obtained using OUCS3 spatial discretization with Euler time-integration scheme after $2000 \Delta t(\Delta t=0.0569)$. Disturbance quantities shown in (i)-(iii): density; (iv)-(vi): pressure and (vii)-(ix): speed. Figure in: (i), (iv) and (vii) are with 5 pt. stencil and two layers of ghost cells; (ii), (v) and (viii) are with 7 pt. stencil and three layers of ghost cells and (iii), (vi) and (ix) are with 9 pt. stencil and four layers of ghost cells.

669 boundary point which we remove by using explicit 4th order dissipation 670 for DNS. However, for the acoustics problem we did not introduce any 671 dissipation. Once again, the RK4 scheme used with the 8th order filter 672 produces results with no oscillations. 

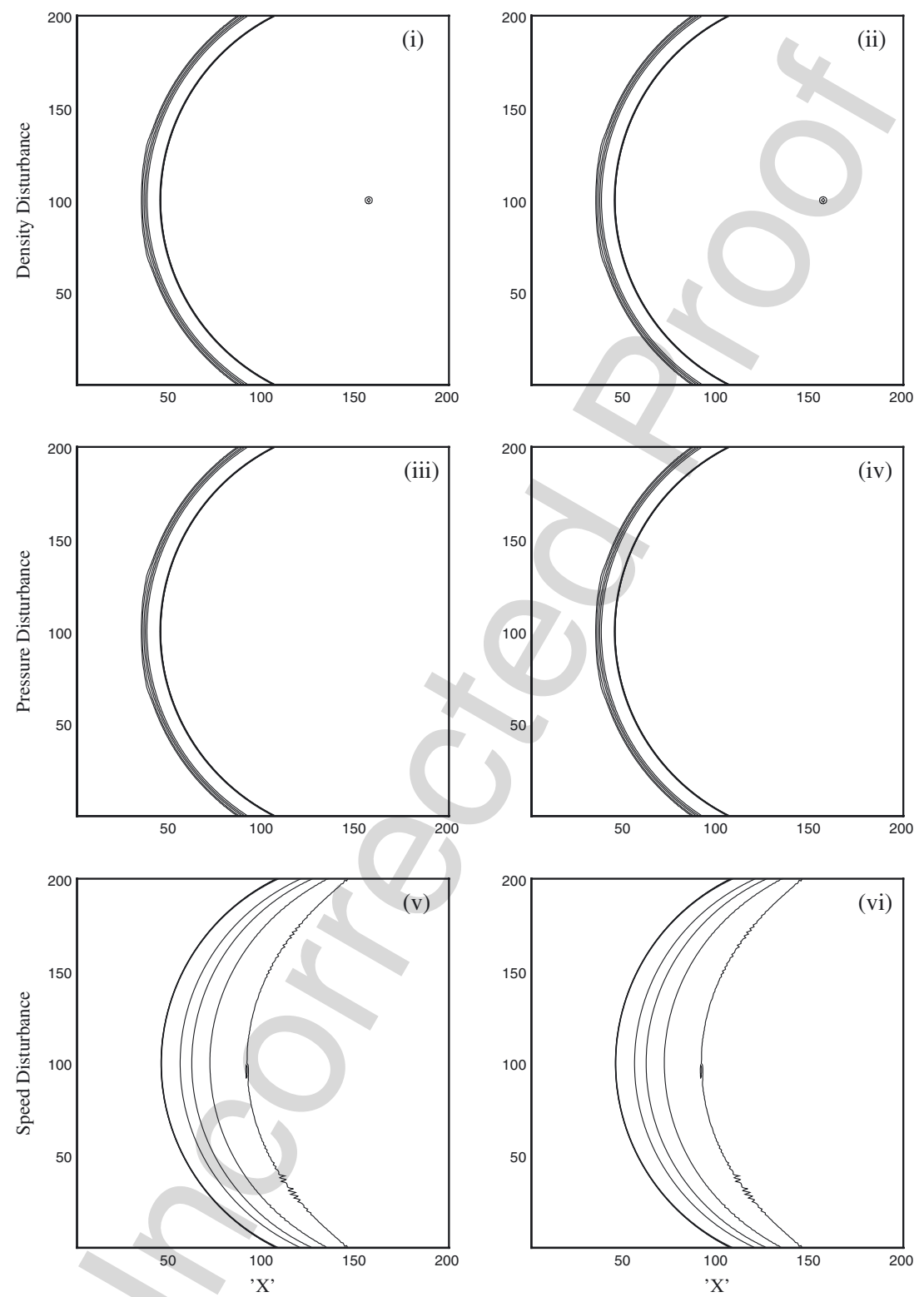

Fig. 17(b). Solution of Eqs. (33) and (34) obtained using OUCS3 spatial discretization with RK4 time-integration scheme after 2000 $\Delta t(\Delta t=0.0569)$. Disturbance quantities shown in (i), (ii): density; (iii), (iv): pressure and (v), (vi): speed. Figures in: (i), (iii) and (v) are with 7 pt. stencil and 3 layers of ghost cells; (ii), (iv) and (vi) are with 9 pt. stencil and 4 layers of ghost cells. 

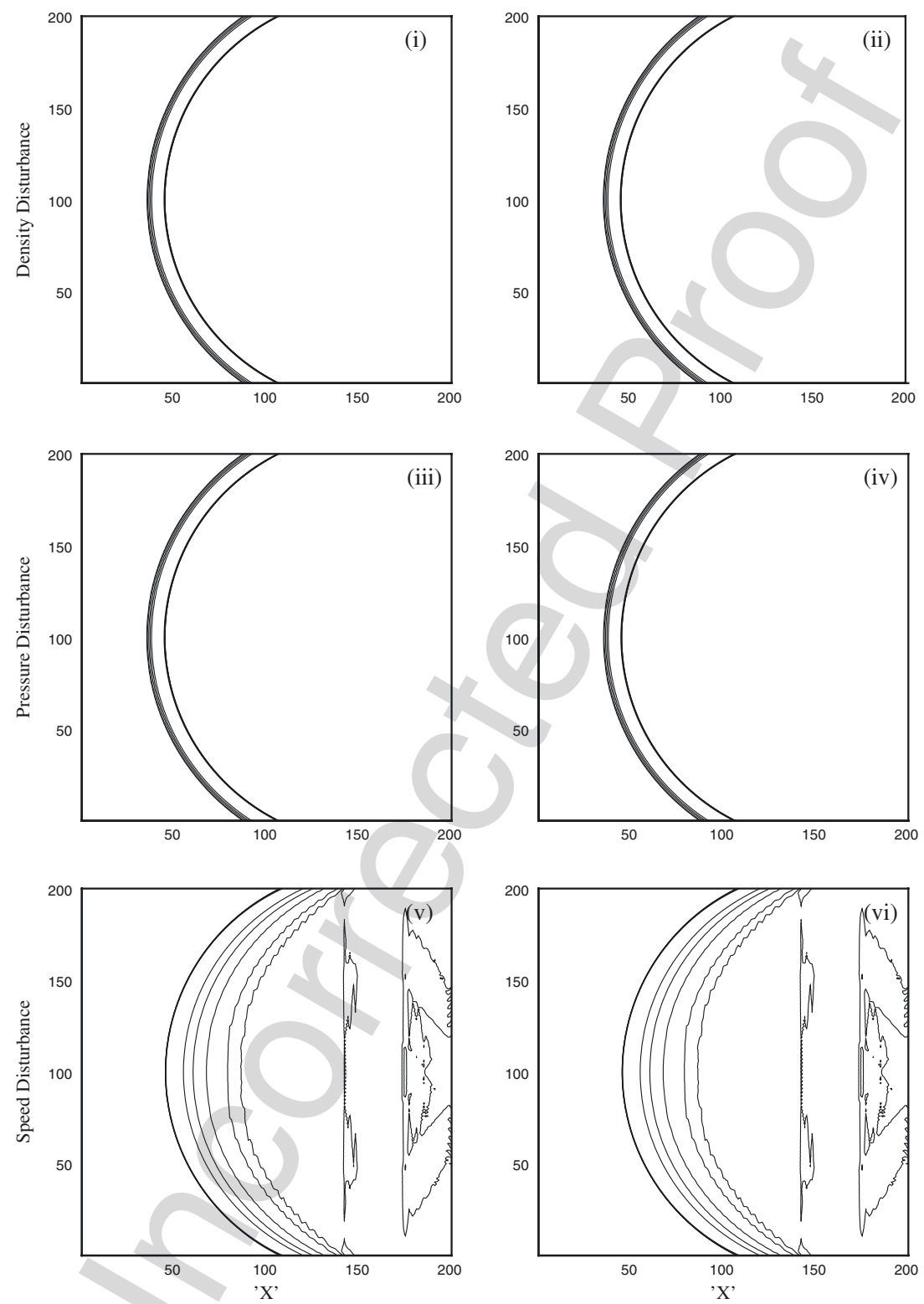

Fig. 17(c). Solution of Eqs. (33) and (34) obtained using OUCS3 spatial discretization with AB time-integration scheme after $2000 \Delta t(\Delta t=0.0569)$. Disturbance quantities shown in (i), (ii): density; (iii), (iv): pressure and (v), (vi): speed. Figure in: (i), (iii) and (v) are with 7 pt. stencil and three layers of ghost cells; (ii), (iv) and (vi) are with 9 pt. stencil and four layers of ghost cells. 

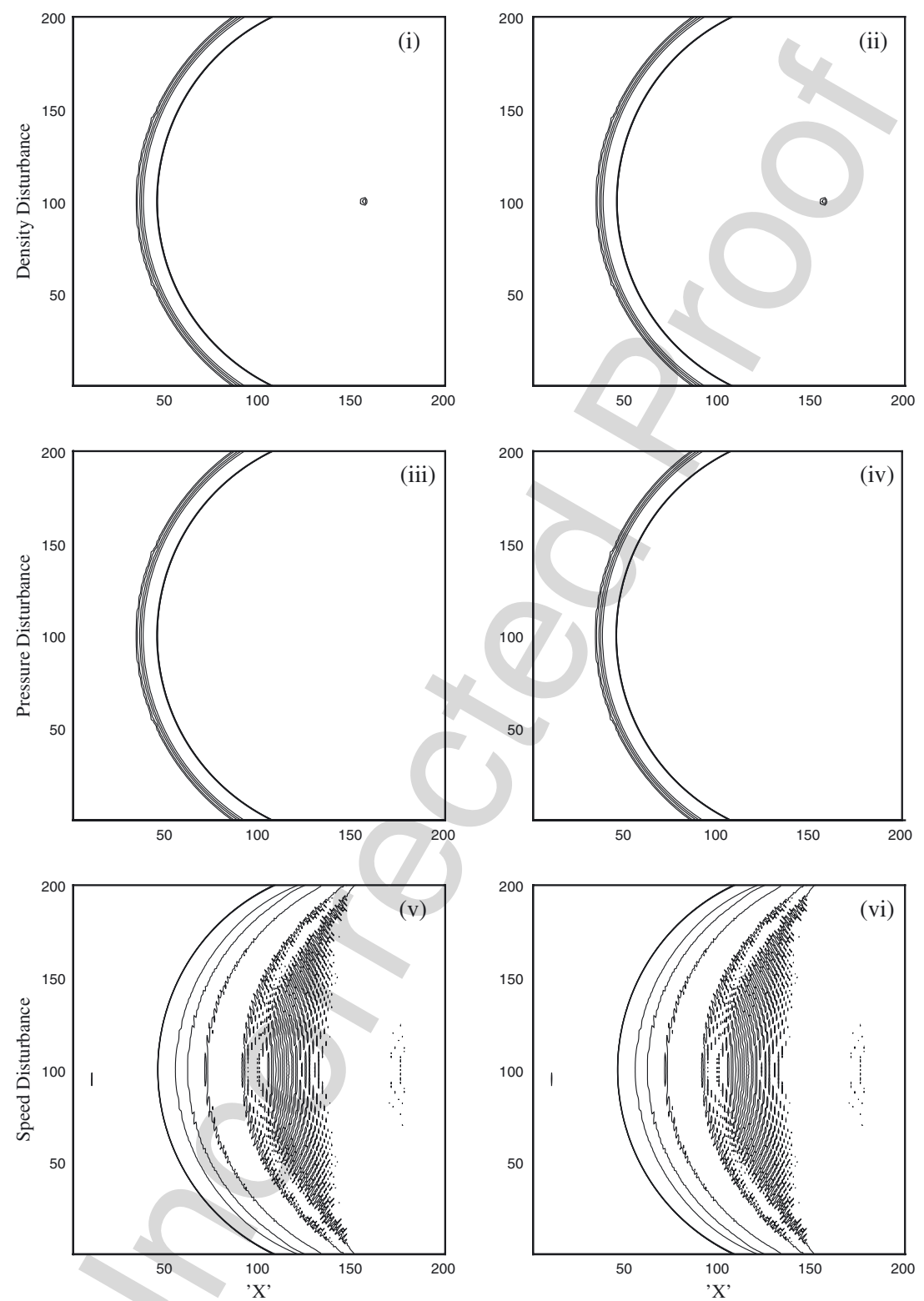

Fig. 18(a). Solution of Eqs. (33) and (34) obtained using OUCS4 spatial discretization with Euler time integration scheme after 2000 $\Delta t(\Delta t=0.0569)$. Disturbance quantities shown in (i), (ii): density; (iii), (iv): pressure and (v), (vi): speed. Figure in: (i), (iii) and (v) are with 7 pt. stencil and three layers of ghost cells; (ii), (iv) and (vi) are with 9 pt. stencil and four layers of ghost cells. 

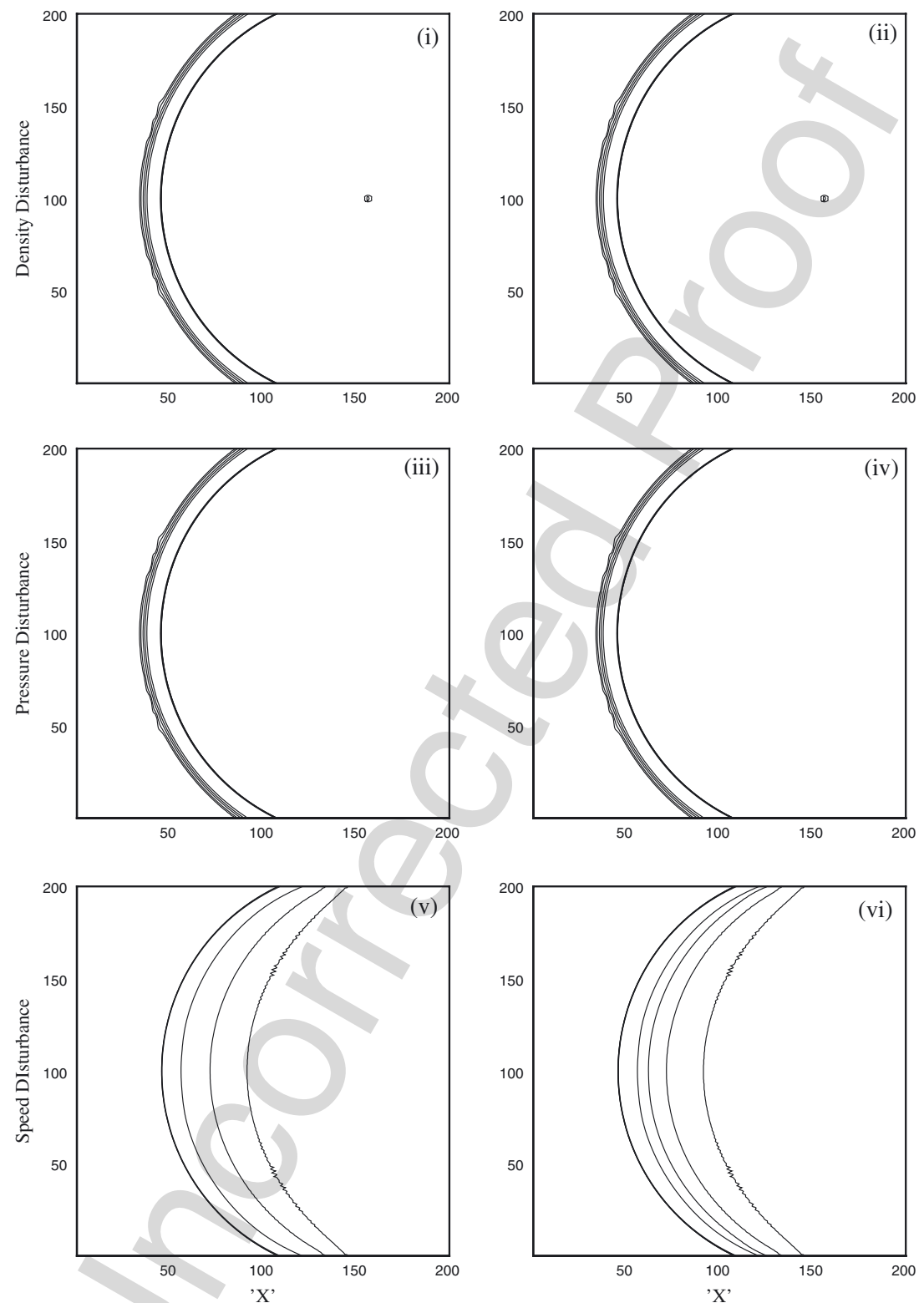

Fig. 18(b). Solution of Eqs. (33) and (34) obtained using OUCS4 spatial discretization with RK4 time integration scheme after 2000 $\Delta t(\Delta t=0.0569)$. Disturbance quantities shown in (i), (ii): density; (iii), (iv): pressure and (v), (vi): speed. Figure in: (i), (iii) and (v) are with 7 pt. stencil and three layers of ghost cells; (ii), (iv) and (vi) are with 9 pt. stencil and four layers of ghost cells. 

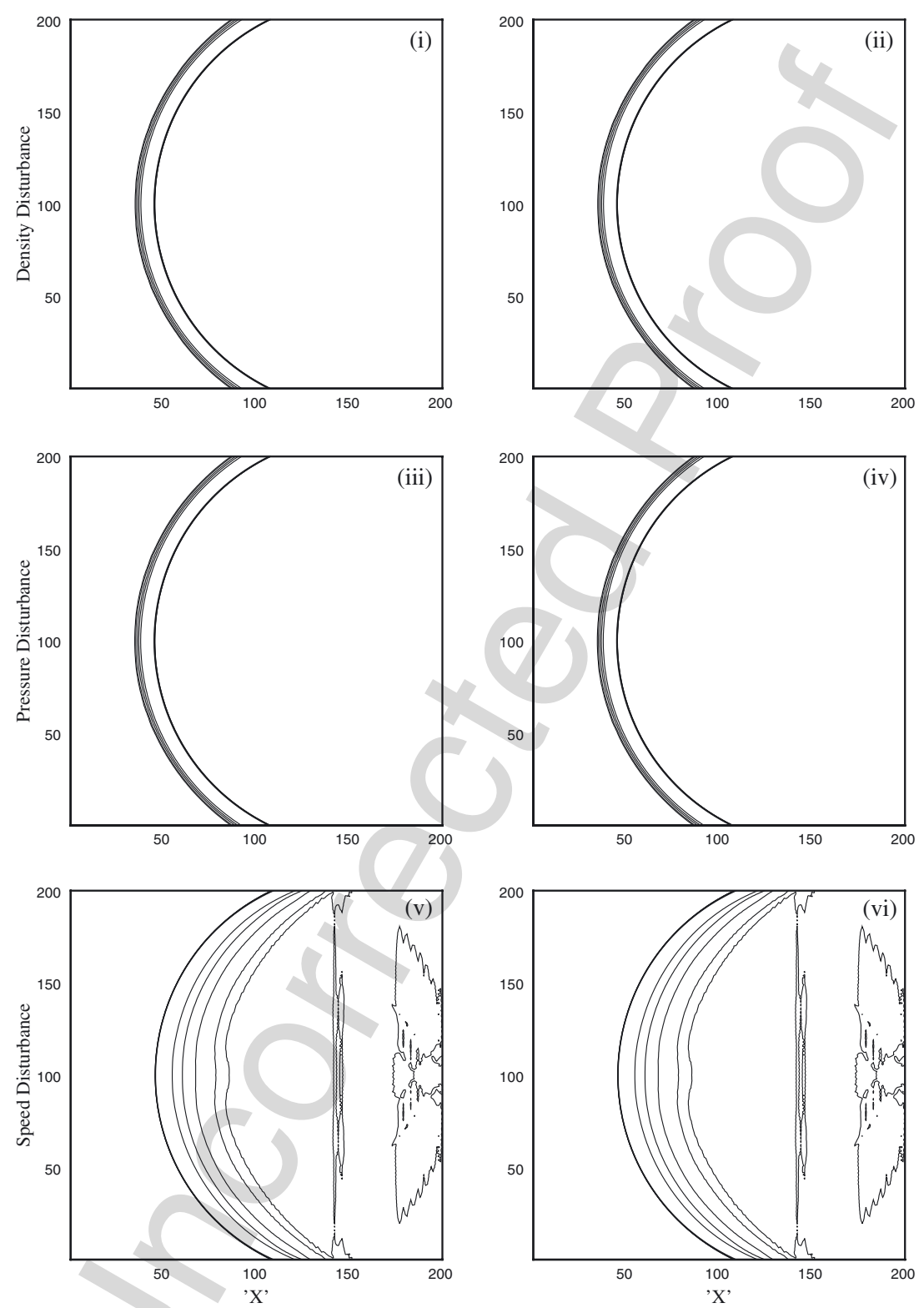

Fig. 18(c). Solution of Eqs. (33) and (34) obtained using OUCS4 spatial discretization with $\mathrm{AB}$ time integration scheme after $2000 \Delta t(\Delta t=0.0569)$. Disturbance quantities shown in (i), (ii): density; (iii), (iv): pressure and (v), (vi): speed. Figures in: (i), (iii) and (v) are with 7 pt. stencil and three layers of ghost cells; (ii), (iv) and (vi) are with 9 pt. stencil and four layers of ghost cells. 


\section{CONCLUSION}

674

675

676

677

678

679

680

681

682

683

684

685

686

687

688

689

690

691

692

693

694

695

696

697

698

699

700

701

702

703

704

705

706

707

708

709

710

711

712

In the present work, we have analyzed some time integration schemes with different high accuracy compact and explicit spatial discretization schemes. Various important properties of spectral accuracy, numerical stability and DRP are investigated. Two examples from DNS and computational acoustics have been solved to highlight the efficacy of various schemes.

Since the major problem in acoustics is to predict weak signals and distinguish it from background noise (which arises due to numerical errors), emphasis is on highly accurate discretization schemes. It is shown that high order does not necessarily imply high accuracy for both space and time schemes. Used spatial compact schemes like the OUCS3 and OUCS4 schemes are only 2nd order formally accurate, and yet they performed as well as the optimum 4th order scheme of [9] and a 6th order accurate optimized explicit scheme (SS scheme) developed by us. The compact schemes has better DRP property than the explicit schemes. We have also investigated the effect of using multi-layer ghost cells at the boundary to avoid spurious reflection, through the SS scheme and its one sided variants. Additionally, higher order filter is used for the same purpose.

Similarly, among time integration schemes, the 2nd order accurate AB-scheme performed poorly compared to 1st order accurate Euler time integration scheme as shown by comparing the numerical results with exact solution of the linearized Euler equation. This is shown due to the presence of a spurious computational mode that is heavily damped and that apportions to itself a large fraction of the initial condition. The 4th accurate RK4 scheme does not suffer from this problem, as this does not have computational mode.

Solution of incompressible Navier-Stokes equation for the problem of a rotating and translating circular cylinder showed physical temporal instability when Euler time integration scheme was used. The AB-scheme could not capture the sharp changes in loads during the instabilities. Solution of compressible Euler equation for an acoustics problem showed, once again, the inadequacy of the AB-scheme. The Euler and RK4 scheme performed satisfactorily, when the latter was used with a 8th order filter to avoid spurious reflection from the boundary.

\section{REFERENCES}

1. Lighthill, M. J. (1993). Computational Aeroacoustics, Springer Verlag, New York.

2. Hirsch, R. S. (1975). J. Comput. Phys. 19, 90.

3. Adam, Y. (1977). J. Comput. Phys. 24, 19.

4. Lele, S. K. (1992). J. Comput. Phys. 103, 16. 
5. Zhong, X. (1998). J. Comput. Phys. 144, 662.

714 6. Adams, N. A. and Shariff, K. (1996). J. Comput. Phys. 127(1), 27.

7. Sengupta, T. K., Ganeriwal, G., De, S. (2003). J. Comp. Phys. 192(2), 677.

716 8. Visbal, M. R. and Gaitonde, D. V. (1999. AIAA J. 37, 1231.

9. Tam, C. K. W. and Webb, J. C. (1993). J. Comput. Phys. 107, 262.

10. Haras, Z. and Ta'asan, S. (1994). J. Comput. Phys. 114, 265.

11. Oran, E. S. and Boris, J. P. (2001). Numerical Simulation of Reactive Flows Cambridge University Press, 2nd Edition, Cambridge, U.K.

12. Durran, D. R. (1999). Numerical Methods for Wave Equation in Geophysical Fluid Dynamics, Springer Verlag, New York.

13. Haltiner, G. J. and Williams, R. T. (1980). Numerical Prediction and Dynamic Meteorology Wiley, New York.

14. Lilly, D. K. (1965). Monthly Weather Review, 138, 11.

15. Kim, J. and Moin, P. (1985). J. Comput. Phys. 58, 308.

16. Kawamura, H., Abe, H. and Matsuo, Y. (1999). Int. J. Heat Fluid Flow. 20, 196.

17. Maass, C. and Schumann, U. (1994). In Direct and Large Eddy Simulation I, (Voke, Kleiser and Chollet), 287.

18. Boersma, B. J., Brethonwer, G. and Nieuwstadt, F. T. M. (1998). Phys. Fluids, 10, 899.

19. Serre, E., Bontoux, P. and Kotorba, R. (2001). Int. J. Fluid Dynamics 5(2), 17.

20. Shi, J., Thomas, T. G. and Williams, J. J. R. (2000). J. Hydraulics Res. 38(6), 465.

733 21. Karamanos, G. S. and Karniadakis, G. E. (2000). J. Comput. Phys. 163, 22.

734 22. Clerex, H. J. H. (1997). J. Comput. Phys. 136, 186.

23. Box, G. E. P. and Draper, N. R. (1969). Evolutionary Operation, Wiley, New York.

24. Sengupta, T. K. and Nair, M. T. (1999). Int. J. Numer. Meth. Fluids. 31, 879.

25. Werle, H. (1984). La Recherche Aérospatiale 1984-4, 39. 